\title{
HUBBLE SPACE TELESCOPE MORPHOLOGIES OF LOCAL LYMAN BREAK GALAXY ANALOGS. I. EVIDENCE FOR STARBURSTS TRIGGERED BY MERGING
}

\author{
Roderik A. Overzier, ${ }^{1,2}$ Timothy M. Heckman, ${ }^{1}$ Guinevere Kauffmann, ${ }^{2}$ Mark Seibert, ${ }^{3}$ R. Michael Rich, ${ }^{4}$ \\ Antara Basu-Zych, ${ }^{5}$ Jennifer Lotz, ${ }^{6}$ Alessandra Aloisi, ${ }^{7}$ Stéphane Charlot, ${ }^{8}$ C. Hoopes, ${ }^{1}$ \\ D. Christopher Martin, ${ }^{9}$ David Schiminovich, ${ }^{5}$ and Barry Madore ${ }^{3}$ \\ Received 2007 September 18; accepted 2007 December 21
}

\begin{abstract}
Heckman and coworkers used the GALEX UV imaging survey to show that there exists a rare population of nearby compact UV-luminous galaxies (UVLGs) that closely resemble high-redshift Lyman break galaxies (LBGs). We present HST images in the UV, optical, and $\mathrm{H} \alpha$ and resimulate them at the depth and resolution of the GOODS/UDF fields to show that the morphologies of UVLGs are also similar to those of LBGs. Our sample of eight LBG analogs thus provides detailed insight into the connection between star formation and LBG morphology. Faint tidal features or companions can be seen in all of the rest-frame optical images, suggesting that the starbursts are the result of a merger or interaction. The UV/optical light is dominated by unresolved $(\sim 100-300 \mathrm{pc})$ super starburst regions (SSBs). A detailed comparison with the galaxies Haro 11 and VV 114 at $z=0.02$ indicates that the SSBs themselves consist of diffuse stars and (super) star clusters. The structural features revealed by the new HST images occur on very small physical scales and are thus not detectable in images of high-redshift LBGs, except in a few cases where they are magnified by gravitational lensing. We propose, therefore, that LBGs are mergers of gas-rich, relatively low-mass $\left(M_{*} \sim 10^{10} M_{\odot}\right)$ systems, and that the mergers trigger the formation of SSBs. If galaxies at high redshifts are dominated by SSBs, then the faint-end slope of the luminosity function is predicted to have slope $\alpha \sim 2$. Our results are the most direct confirmation to date of models that predict that the main mode of star formation in the early universe was highly collisional.
\end{abstract}

Subject headings: cosmology: observations — early universe — galaxies: high-redshift — galaxies: starburst

\section{INTRODUCTION}

How did galaxies form? Ultimately, this simple question captures most, if not all, of the most widely pursued research topics in cosmology. Surveys of galaxies at different redshifts are being used to study which galaxies contain most of the stellar mass at a given epoch, and which galaxies are undergoing the strongest evolution. These measurements serve to constrain models of structure formation. The main formative processes associated with typical galaxies (e.g., star formation, merging, and feedback) were largely completed within the first half of the Hubble time, making their study a very challenging one. One of the best probes for studying star formation in the early universe is provided by galaxies that are luminous in the rest-frame UV due to intense star formation. The most luminous of these, the Lyman break galaxies (LBGs), are easily detected at $z=2-6$ in deep pencil beam surveys from the ground and with the Hubble Space Telescope (HST; e.g., Steidel \& Hamilton 1993; Steidel et al. 1999; Shapley et al. 2001; Giavalisco et al. 2004; Adelberger et al. 2004; Bouwens

\footnotetext{
1 Department of Physics and Astronomy, Johns Hopkins University, Baltimore, MD 21218.

${ }^{2}$ Max-Planck-Institut für Astrophysik, D-85748 Garching, Germany; overzier@ mpa-garching.mpg.de.

3 Observatories of the Carnegie Institution of Washington, Pasadena, CA 91101.

4 Department of Physics and Astronomy, Division of Astronomy and Astrophysics, University of California, Los Angeles, CA 90095-1562.

5 Department of Astronomy, Columbia University, New York, NY 10027.

6 Department of Astronomy and Astrophysics, University of California, Santa Cruz, CA 95064.

7 Space Telescope Science Institute, Baltimore, MD 21218.

8 Institut d'Astrophysique du CNRS, F-75014 Paris, France.

9 California Institute of Technology, Pasadena, CA 91125.
}

et al. 2006; Yoshida et al. 2006). The cosmic star formation rate (SFR) reached a maximum at $z \sim 1-3$ and decreased dramatically toward $z=0$ (e.g., Lilly et al. 1996; Schiminovich et al. 2005). There is only a modest decrease in the UV luminosity density from $z \sim 3$ to $z \sim 6$ (e.g., Ouchi et al. 2004a; Bouwens et al. 2006), indicating that LBGs represent a major phase in the early stages of galaxy formation and evolution. Based on their clustering and number statistics, LBGs are the precursors of presentday massive galaxies undergoing a phase of intense star formation, and a large fraction of LBGs may merge to form elliptical galaxies that are situated in present-day groups and clusters (Governato et al. 2001; Giavalisco \& Dickinson 2001; Moustakas \& Somerville 2002; Ouchi et al. 2004b; Adelberger et al. 2005).

Studies of the structure and sizes of LBGs indicate that high-redshift galaxies are compact $\left(\sim 0.1^{\prime \prime}-0.3^{\prime \prime}\right.$, or $\sim 1-2.5 \mathrm{kpc}$; Giavalisco et al. 1996b; Lowenthal et al. 1997; Ferguson et al. 2004; Bouwens et al. 2004) and that large ( $\left.\gtrsim 0.4^{\prime \prime}\right)$ low surface brightness galaxies are rare. The morphologies are best characterized as being dominated by one to several UV-bright knots embedded in diffuse emission, with little difference between the rest-frame UV and optical light distributions (Papovich et al. 2001, 2005). The morphologies of LBGs are unlike those of nearby galaxies on the Hubble sequence. This is true even if the images of the nearby objects are degraded so that they have similar resolution and depth to those of LBGs. LBG morphologies are more similar to those of ultraluminous infrared galaxies (ULIRGs), which occupy a region of the concentration/asymmetry plane that lies between double-nucleated mergers, spheroids, and disks (e.g., Abraham et al. 1996; Conselice et al. 2004; Lotz et al. 2004, 2006; Law et al. 2007b and references therein). However, the interpretation of the UV morphologies of high-redshift populations remains problematic (see Law et al. 2007b) because it is unclear 
whether the irregular morphologies are a consequence of the merging of gas-rich galaxies, or whether one is just seeing patchily distributed star formation within a single system. The answer to this question and how it evolves with redshift is essential for understanding how galaxies formed.

A detailed study of LBGs is limited by two factors. First, their distances render them small and faint, and, second, star-forming galaxies at high redshift are systematically different from starburst galaxies in the nearby universe. Local starbursts (SBs) show strong correlations among their basic properties that are different from LBGs. In particular, there is a systematic increase in the amount of dust obscuration $\left(L_{\mathrm{FIR}} / L_{\mathrm{FUV}}\right)$ with increasing SFR, corresponding to a systematic increase in metallicity with mass. LBGs are substantially less obscured than local SBs of similar SFR or mass (e.g., Reddy et al. 2006). The relatively low extinction of LBGs is also a characteristic of blue compact dwarfs (BCDs, or $\mathrm{H}$ II galaxies), but the latter have SFRs that are typically 2 orders of magnitude smaller than LBGs and have lower masses (e.g., Fanelli et al. 1988; Telles \& Terlevich 1997; Hunter \& Hoffman 1999; Gil de Paz et al. 2003). Although BCDs may resemble the faintest LBGs (e.g., Meurer et al. 1995; Noeske et al. 2006) and LIRGs and ULIRGs may resemble the dustiest LBGs (e.g., Adelberger \& Steidel 2000; Goldader et al. 2002; Daddi et al. 2005; Chapman et al. 2005; Huang et al. 2005; van Dokkum et al. 2006), typical local SBs are different from typical LBGs. LBGs have systematically lower metallicities and higher gas mass fractions than local SBs with the same SFRs. The typical mechanisms that trigger starbursts might also be very different at high redshift compared to low redshift.

Clearly, if we could find relatively nearby starburst galaxies whose properties are the best match to LBGs, we could study for the first time at a much higher physical resolution how the vigorous star formation and morphologies of typical high-redshift galaxies are related. However, local LBG analogs are currently very rare; as indicated by the Galaxy Evolution Explorer (GALEX; Martin et al. 2005) imaging survey in the near-ultraviolet (NUV; $\lambda \sim 2250 \AA$ ) and far-ultraviolet (FUV; $\lambda \sim 1550 \AA$ ), the comoving number density of galaxies with FUV luminosities similar to LBGs has declined by a factor of several hundred between $z=3$ and 0. Heckman et al. (2005, hereafter H05) and Hoopes et al. (2007, hereafter H07) identified a rare population of low-redshift $(z<0.3)$ galaxies with properties remarkably similar to those of LBGs by matching sources in the GALEX all-sky survey with the Sloan Digital Sky Survey (SDSS; York et al. 2000) spectroscopic sample and selecting according to two criteria specifically designed to match the typical UV properties of LBGs (e.g., Steidel et al. 1996; Shapley et al. 2001): $L_{\mathrm{FUV}} \geq 10^{10.3} L_{\odot} \wedge I_{\mathrm{FUV}} \geq$ $10^{9} L_{\odot} \mathrm{kpc}^{-2}$, where $L_{\mathrm{FUV}}$ is the FUV luminosity $\left(\lambda P_{\lambda}\right)$ and $I_{\mathrm{FUV}}$ is the mean FUV surface brightness interior to the SDSS $u$-band half-light radius $\left(I_{\mathrm{FUV}}=\frac{1}{2} L_{\mathrm{FUV}} / \pi r_{e, u}^{2}\right)$. By further limiting the sample to $z<0.3$ and excluding broad-line active galactic nuclei (AGNs), this resulted in a sample of 31 "supercompact UV-luminous galaxies" (UVLGs). Interestingly, once selected according to these criteria, the UVLG sample proved to be similar to typical LBGs in all other measurable properties (see H05 and H07). Further details on the sample are given by Basu-Zych et al. (2007), who investigated the radio to UV continuum properties of the sample and found that they follow the radio-far-infrared correlation of normal star-forming galaxies but generally have less dust than other star-forming galaxies with such high specific SFRs. In this respect, they are similar to LBGs. This suggests that the process that causes star formation in the supercompact UVLGs differs from other local star-forming galaxies but may be similar to LBGs.
Our sample of UVLGs ("local LBG analogs") is therefore highly valuable for understanding the nature of LBGs at high redshifts. ${ }^{10}$

In this paper (Paper I) we present the first set of high-resolution HST images of eight UVLGs and discuss their morphologies. In a subsequent paper (Paper II) we will carry out a more detailed study using a large HST data set to be observed in Cycle 16. The structure of this paper is as follows. In $\S 2$ we describe the new observations with $H S T$. In $\S \S 3$ and 4 we present the data, investigate UVLG morphologies both qualitatively and quantitatively, resimulate our data at higher redshift, and compare with morphologies of star-forming galaxies and LBGs, as well as two of the most nearby LBG-like galaxies known (Haro 11 and VV 114). We discuss the implications of our results for understanding the nature of LBGs at high redshifts in $\S 5$, followed by a summary of the results $(\S 6)$. We use a cosmology $\left(\Omega_{M}, \Omega_{\Lambda}, h\right)=(0.27$, $0.73,0.73)$ with $H_{0}=100 h \mathrm{~km} \mathrm{~s}^{-1} \mathrm{Mpc}^{-1}$.

\section{DATA}

\subsection{HST Observations}

We have observed eight of the nearest $(0.091<z<0.204)$ and brightest local LBG analogs of H05 and H07 with HST in Cycle 15. To date, seven UVLGs have been observed with the Advanced Camera for Surveys (ACS) High Resolution Camera (HRC) in the filter F330W and with the Wide Field Channel (WFC) through the filter F850LP. In addition, one UVLG has been observed with the Solar Blind Channel (SBC) in the filter F150LP and with the Wide Field and Planetary Camera 2 (WFPC2) through F606W, given the new constraints following the failure of ACS during the course of 2007. Ramp filter images with a central wavelength equal to that of redshifted $\mathrm{H} \alpha$ were also obtained for all but the latter source. Each filter probes star formation and morphology at a different characteristic timescale. The F330W filter (central wavelength $\lambda_{c} \approx 3334 \AA$ with an effective width $\Delta \lambda \sim 548 \AA$ ) is the most sensitive to star formation over the past $\sim 100 \mathrm{Myr}$ (Leitherer \& Heckman 1995) and provides the closest match to the GALEX NUV filter $\left(\lambda_{c} \approx 2315 \AA, \Delta \lambda \sim 730 \AA\right)$. The F150LP filter $\left(\lambda_{c} \approx 1614 \AA, \Delta \lambda \sim 234 \AA\right)$, available for one of the UVLGs, provides an excellent match to the GALEX FUV filter $\left(\lambda_{c} \approx 1530 \AA, \Delta \lambda \sim 255 \AA\right)$. The F606W $\left(\lambda_{c} \approx 6001 \AA\right)$ and F850LP $\left(\lambda_{c} \approx 9170 \AA\right)$ images are well matched to the SDSS images in the $r$ and $i$ band and probe older ( $>$ Gyr) stellar populations. The ACS ramp filters (FR647M with a width of $207 \AA$ or FR782N with a width of $52 \AA$, depending on the redshift) probe redshifted $\mathrm{H} \alpha$ from $\mathrm{H}$ II regions tracing the youngest stellar population (O stars with lifetimes $<10 \mathrm{Myr}$ ) and allow us to search for evidence of galaxy-wide outflows of ionized gas.

The targets were observed for one orbit per filter with ACS and two orbits per filter with WFPC2. The F330W image of SDSS J092600.41+442736.1 failed to execute due to a guide star problem. The F150LP, F330W, ramp filter, F606W, and F850LP images consisted of three, three, two, six, and three exposures, respectively, to facilitate the removal of cosmic rays. The images were combined using multidrizzle (Koekemoer et al. 2002), producing registered, cosmic-ray-free, geometrically corrected images. The SBC and HRC images have a plate scale of $0.025^{\prime \prime}$ pixel $^{-1}$ and a resolution of $\sim 0.07^{\prime \prime}$ (FWHM). The WFPC2/PC images have a plate scale of $0.046^{\prime \prime}$ pixel $^{-1}$ with a resolution of $\approx 0.11^{\prime \prime}$ (FWHM). The WFC images have a scale of $0.05^{\prime \prime}$ pixel $^{-1}$ with a resolution

\footnotetext{
10 See the Appendix for a detailed analysis that demonstrates that the interpretation and conclusions presented in the recent paper by Scarpa et al. (2007) are in fact in error.
} 
TABLE 1

Sample and Log of HST ACS Observations

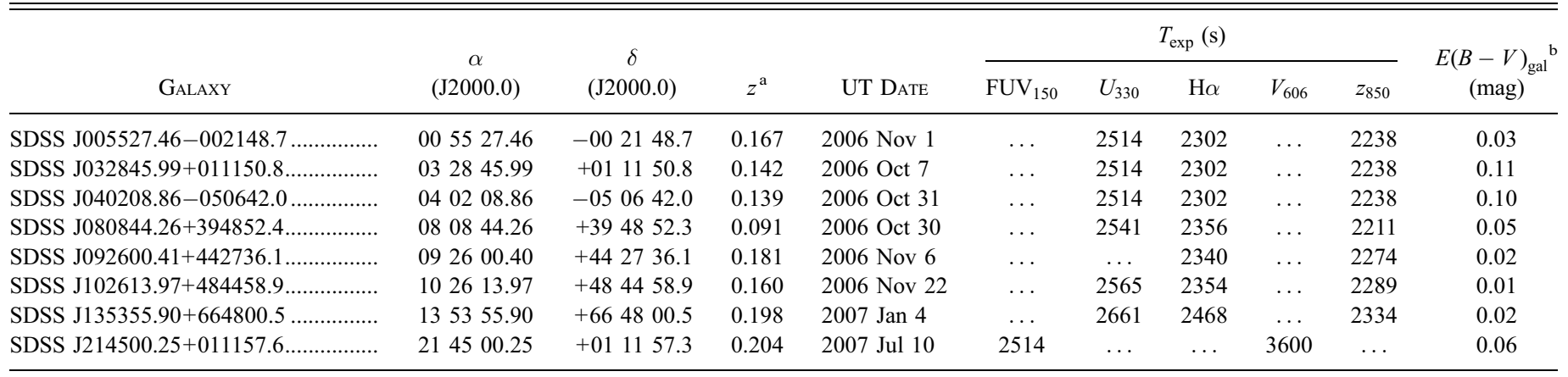

Nоте.-Units of right ascension are hours, minutes, and seconds, and units of declination are degrees, arcminutes, and arcseconds.

${ }^{\text {a }}$ Spectroscopic redshifts from SDSS.

b Galactic extinction as given by Schlegel et al. (1998).

of $\approx 0.12^{\prime \prime}(\mathrm{FWHM})$. Magnitudes in the AB system were measured using SExtractor (Bertin \& Arnouts 1996) from $m_{\mathrm{AB}}=$ $-2.5 \log _{10}$ (counts $\left./ T_{\exp }\right)+\mathrm{ZP}$, where the zero points ( $\left.\mathrm{ZPs}\right)$ are 22.448, 24.085, 23.004, and $24.862 \mathrm{mag}$ for F150LP, F330W, F606W, and F850LP, respectively. All magnitudes were corrected for Galactic extinction using the dust maps of Schlegel et al. (1998). The reader is referred to Table 1 for a log of the observations, Table 2 for SFRs and stellar masses of the objects in our sample, and Table 3 for the main photometric data from GALEX and ACS.

\subsection{Continuum-subtracted $\mathrm{H} \alpha$ Images}

The $\mathrm{H} \alpha$ ramp filter images were continuum subtracted as follows. First, we scaled the ACS $z_{850}$ image to an artificial $i_{775}$ continuum image, by determining the ratio of the flux of the $i_{775}$ continuum at the wavelength of redshifted $\mathrm{H} \alpha$ (measured from the SDSS fiber spectrum on opposite sides of the $\mathrm{H} \alpha$ line) to the total flux in the $z_{850}$ image. The total flux was measured within an aperture similar in size to the $3^{\prime \prime}$ diameter SDSS fiber aperture. We then created a "continuum-free" $\mathrm{H} \alpha$ image by subtracting the artificial $i_{775}$ continuum image from the ACS ramp filter image. The resulting $\mathrm{H} \alpha$ image should be a very good approximation, under the (very reasonable) assumption that the morphology of the continuum does not change between $i_{775}$ and $z_{850}$. In the case of object SDSS J080844.26+394852.4 the subtraction was problematic due to the fact that it relied on subtraction of two bright point sources in $z_{850}$ and $\mathrm{H} \alpha$.

\subsection{Spitzer Observations}

Infrared photometry was obtained with the Infrared Array Camera (IRAC) and the Multiband Imaging Photometer for Spitzer (MIPS) aboard the Spitzer Space Telescope (PI: C. Hoopes, No. 20390). The total integration time with IRAC at 3.6, 4.5, 5.8, and $8.0 \mu \mathrm{m}$ was $60 \mathrm{~s}$ for each channel. The total integration time with MIPS at 24 and $70 \mu \mathrm{m}$ was 42 and $60 \mathrm{~s}$, respectively. We used a minimum five-step dither pattern for removal of cosmic rays. The post-basic calibrated data delivered by the pipeline were used to measure the integrated flux densities. The infrared photometry is given in Table 5 below.

\subsection{Comparison Data}

The UVLGs in our sample, which have a median redshift of $z \sim 0.15$, lie at redshifts that are intermediate between local starburst galaxies $(\sim 0.02)$ and LBGs at high redshift $(z>1.5)$. Throughout the paper, we therefore find it instructive to present our results with respect to the following two sets of comparison data.

\subsubsection{Archival Imaging Data of Haro 11 and VV 114}

As shown in $\mathrm{H} 07$ (see their Fig. 12), a number of very nearby blue compact starburst galaxies and (U)LIRGs fall very near to the boundary of the UVLG selection window as defined in the $L_{\mathrm{FUV}}$ vs. $I_{\mathrm{FUV}}$ plane. Because there is quite some leverage in the definition of a typical "LBG," we compare our data to two of

TABLE 2

Star Formation Rates and Stellar Masses

\begin{tabular}{|c|c|c|c|c|}
\hline Galaxy & $\begin{array}{c}\mathrm{SFR}_{\mathrm{FUV}}{ }^{\mathrm{a}} \\
\left(M_{\odot} \mathrm{yr}^{-1}\right)\end{array}$ & $\begin{array}{c}\mathrm{SFR}_{\mathrm{IR}^{\mathrm{b}}} \\
\left(M_{\odot} \mathrm{yr}^{-1}\right)\end{array}$ & $\begin{array}{l}\mathrm{SFR}_{1.4 \mathrm{GHz}}{ }^{\mathrm{a}} \\
\left(M_{\odot} \mathrm{yr}^{-1}\right)\end{array}$ & $\begin{array}{c}M_{*}{ }^{\mathrm{c}} \\
\left(10^{10} M_{\odot}\right)\end{array}$ \\
\hline SDSS J005527.46-002148.7 ……………...... & $4.2 \pm 0.8$ & 28 & $14.9 \pm 2.0$ & 1.17 \\
\hline SDSS J032845.99+011150.8....................... & $3.2 \pm 1.1$ & $<3.4$ & $<4.6$ & 0.55 \\
\hline SDSS J040208.86-050642.0 ........................ & $3.7 \pm 0.6$ & $<2.8$ & $<4.1$ & 0.25 \\
\hline SDSS J080844.26+394852.4........................ & $3.1 \pm 0.4$ & 7.3 & $<3.7$ & 2.14 \\
\hline SDSS J092600.41+442736.1.......................... & $6.8 \pm 0.4$ & 3.7 & $7.8 \pm 1.4$ & 0.16 \\
\hline SDSS J102613.97+484458.9........................... & $3.4 \pm 0.1$ & 6.6 & $9.2 \pm 1.3$ & 0.81 \\
\hline SDSS J135355.90+664800.5 ……………....... & $7.1 \pm 0.9$ & 14.6 & $22.5 \pm 2.7$ & 0.98 \\
\hline SDSS J214500.25+011157.6 & $3.4 \pm 0.8$ & 7.5 & $14.5 \pm 2.9$ & 1.32 \\
\hline
\end{tabular}

${ }^{a}$ UV SFR derived from the FUV luminosity (uncorrected for dust). Adapted from Basu-Zych et al. (2007).

${ }^{\mathrm{b}}$ IR SFR derived from the bolometric dust luminosity (given in Table 5) and calculated using the formula $\log _{10} L_{\mathrm{dust}}=$ $9.75+\log _{10}$ SFR IR $_{\text {IR }}$ and assuming a 100 Myr old constant SFR model with Salpeter IMF as given in Takeuchi et al. (2005).

${ }^{c}$ Stellar mass based on SED model fitting. Adapted from H07 (see also Salim et al. 2007). 
TABLE 3

Photometry and UV Spectral Slope

\begin{tabular}{|c|c|c|c|c|c|c|}
\hline Galaxy & $\begin{array}{l}m_{\mathrm{FUV}} \\
(\mathrm{mag})\end{array}$ & $\begin{array}{l}m_{\mathrm{NUV}} \\
(\mathrm{mag})\end{array}$ & $\begin{array}{c}m_{330} \\
(\mathrm{mag})\end{array}$ & $\begin{array}{c}m_{u} \\
(\mathrm{mag})\end{array}$ & $\begin{array}{c}m_{850} \\
(\mathrm{mag})\end{array}$ & $\beta_{\text {FUV-NUV }}$ \\
\hline SDSS J005527.46-002148.7 .................. & $19.23_{-0.17}^{+0.15}$ & $18.85_{-0.08}^{+0.08}$ & 18.78 & $18.65 \pm 0.02$ & 18.25 & $-1.13 \pm 0.22$ \\
\hline SDSS J032845.99+011150.8 $\ldots \ldots \ldots \ldots \ldots \ldots \ldots$ & $19.42_{-0.31}^{+0.11}$ & $19.23_{-0.16}^{+0.14}$ & 18.97 & $18.68 \pm 0.04$ & 18.15 & $-1.56 \pm 0.39$ \\
\hline SDSS J040208.86-050642.0 …............. & $18.90_{-0.06}^{+0.06}$ & $18.70_{-0.03}^{+0.03}$ & 18.81 & $18.67 \pm 0.03$ & 18.08 & $-1.54 \pm 0.09$ \\
\hline SDSS J080844.26+394852.4 „................. & $18.22_{-0.11}^{+0.10}$ & $17.59_{-0.05}^{+0.05}$ & 17.62 & $17.48 \pm 0.01$ & 17.05 & $-0.54 \pm 0.15$ \\
\hline SDSS J092600.41+442736.1 .................... & $18.83_{-0.13}^{+0.11}$ & $18.89_{-0.08}^{+0.07}$ & a & $19.04 \pm 0.03$ & 19.28 & $-2.12 \pm 0.18$ \\
\hline 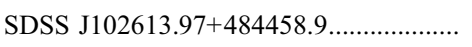 & $19.27_{-0.03}^{+0.13}$ & $19.00_{-0.02}^{+0.08}$ & 18.86 & $18.85 \pm 0.02$ & 18.23 & $-1.38 \pm 0.05$ \\
\hline SDSS J135355.90+664800.5 ….............. & $18.99_{-0.04}^{+0.03}$ & $18.57_{-0.02}^{+0.02}$ & 18.42 & $18.35 \pm 0.02$ & 17.67 & $-1.03 \pm 0.06$ \\
\hline SDSS J214500.25+011157.6 ................... & $19.94_{-0.32}^{+0.04}$ & $19.22_{-0.13}^{+0.02}$ & $19.61^{\mathrm{b}}$ & $19.24 \pm 0.04$ & $18.62^{\mathrm{c}}$ & $-0.35 \pm 0.41$ \\
\hline
\end{tabular}

a We did not obtain data in F330W.

b These measurements correspond to the ACS/SBC F150LP data, instead of ACS/HRC F330W.

c These measurements correspond to the WFPC2 F606W data, instead of ACS/WFC F850LP.

these objects, which have been found to possess some properties that are similar to high-redshift LBGs. The two local comparison objects are Haro 11 and VV 114, both of which lie at $z=0.02$ (for details see Knop et al. 1994; Scoville et al. 2000; Goldader et al. 2002; Bergvall \& Östlin 2002; Kunth et al. 2003; Grimes et al. 2006, 2007 and references therein). Haro 11 is a BCD galaxy consisting of a number of UV-bright knots believed to be in the process of merging. VV 114 is a merging system consisting of a UV-luminous western component (VV 114W) and an IR-luminous eastern component ( VV 114E) with very little associated UV emission.

In this paper we make use of an ACS/HRC image of Haro 11 taken through the filter F220W $\left(\lambda_{c} \approx 2255 \AA\right)$ obtained from the HST archive (Program 10575; PI: G. Östlin). For VV 114(W), we use a Space Telescope Imaging Spectrograph (STIS) image in the NUV $\left(\lambda_{c} \approx 2365 \AA\right)$ from Program 8201 (PI: G. Meurer).

\subsubsection{High-Redshift Samples from Lotz et al. (2006)}

Our high-redshift comparison samples consist of 55 starburst galaxies at $z \sim 1.5$ selected from GOODS and two (largely nonoverlapping) samples of in total $82 z \sim 4$ LBGs in GOODS and the UDF from Lotz et al. (2006). The galaxy sample at $z \sim 1.5$ is based on a large spectroscopic sample of strong emission line galaxies. We used the publicly available GOODS and UDF data and extracted postage stamps of the objects in $B_{435}$ for the $z \sim 1.5$ sample and in $V_{606}+i_{775}$ for the $z \sim 4$ sample. A detailed description of the data and sample selection can be found in Lotz et al. (2006).

\section{RESULTS}

\subsection{Images and Notes on Individual Galaxies}

In Figure 1 we show the GALEX FUV and NUV images. The $\mathrm{UV}, \mathrm{H} \alpha$, and optical images taken with $H S T$ are shown in Figure 2, which have a resolution of $\sim 50$ times that of the GALEX images. Qualitative remarks on the morphologies of the UVLGs in Figure 2 are as follows.

SDSS J005527.46-002148.7.- This object consists of a strong point source surrounded by diffuse emission that can be seen in $U_{330}, z_{850}$, and $\mathrm{H} \alpha$. The diffuse emission has a high surface brightness region extending to the northeast with respect to the nucleus, which suggests either the infall of a small diffuse galaxy or an additional off-center star-forming region. The continuum-subtracted $\mathrm{H} \alpha$ emission is peculiar with an "arm" of emission extending to about $1^{\prime \prime}$ south of the nucleus. The morphology of the $\mathrm{H} \alpha$ is very different from the $z_{850}$ morphology, and at its outer extremity, the $\mathrm{H} \alpha$ is not associated with any UV emission. We suspect that the extended $\mathrm{H} \alpha$ is due to an outflow over the larger region probed by the $z_{850}$ continuum.

$S D S S$ J032845.99+011150.8. - The $z_{850}$ image suggests a merger of two low surface brightness galaxies, as evidenced by the tidal tails extending symmetrically from the center to the northwest and northeast. The starburst dominates the nuclear region, in which five isolated point sources can be seen. The easternmost knot dominates in $\mathrm{H} \alpha$.

SDSS J040208.86-050642.0.- The $z_{850}$ image suggests a merger of two diffuse systems as the contours of faint $z_{850}$ continuum emission show a sharp bend in position angle. The image center is dominated by three point sources, and several fainter ones lie farther out on either side of the center. One of these faint, compact regions (about $1^{\prime \prime}$ south of the nucleus) is more extended in $\mathrm{H} \alpha$.

SDSS J080844.26+394852.4.- There is a bright, unresolved source seen in the $U_{330}$ image that is located in the center of an extended, highly diffuse galaxy seen in $z_{850}$. The unresolved source is remarkably bright as both $U_{330}$ and $\mathrm{H} \alpha$ show the ACS diffraction spikes. The $\mathrm{H} \alpha$ image suffers from poor point-source subtraction. The object seen $\sim 2^{\prime \prime}$ to the south lies at a similar redshift and may be interacting. The nucleus of this companion is also detected in $U_{330}$, but its flux is $\sim 75$ times lower.

SDSS J092600.41+442736.1. - The faint contours of the diffuse emission in $z_{850}$ suggest that at least two diffuse galaxies may be merging. A bright, compact component dominates in $z_{850}$ and $\mathrm{H} \alpha$. The faint extension about $1^{\prime \prime}$ east of the nucleus in $z_{850}$ has little associated $\mathrm{H} \alpha$ emission. The $U_{330}$ image failed to execute due to a guide star problem.

SDSS J102613.97+484458.9.- This galaxy has a ring-shaped morphology with most of the starburst occurring along the eastern rim. There is also an isolated knot to the southwest. The starforming region contains several bright, unresolved components and diffuse emission. A very faint diffuse companion can be seen in $z_{850}$, about $2^{\prime \prime}$ to the south, and very faint extended emission, possibly tidal debris, is seen directly to the east along the entire extent of the galaxy. The overall morphology is very similar to that of the "drop-through" ring galaxy NGC 922 studied by Wong et al. (2006).

SDSS J135355.90+664800.5.- This UVLG is highly irregular in all bands, showing many star-forming knots and diffuse emission. The object is interacting or merging with a warped, edge-on, or filamentary galaxy located just to the east, which is much redder and only just detected in $U_{330}$. A long-slit spectrum along the major 

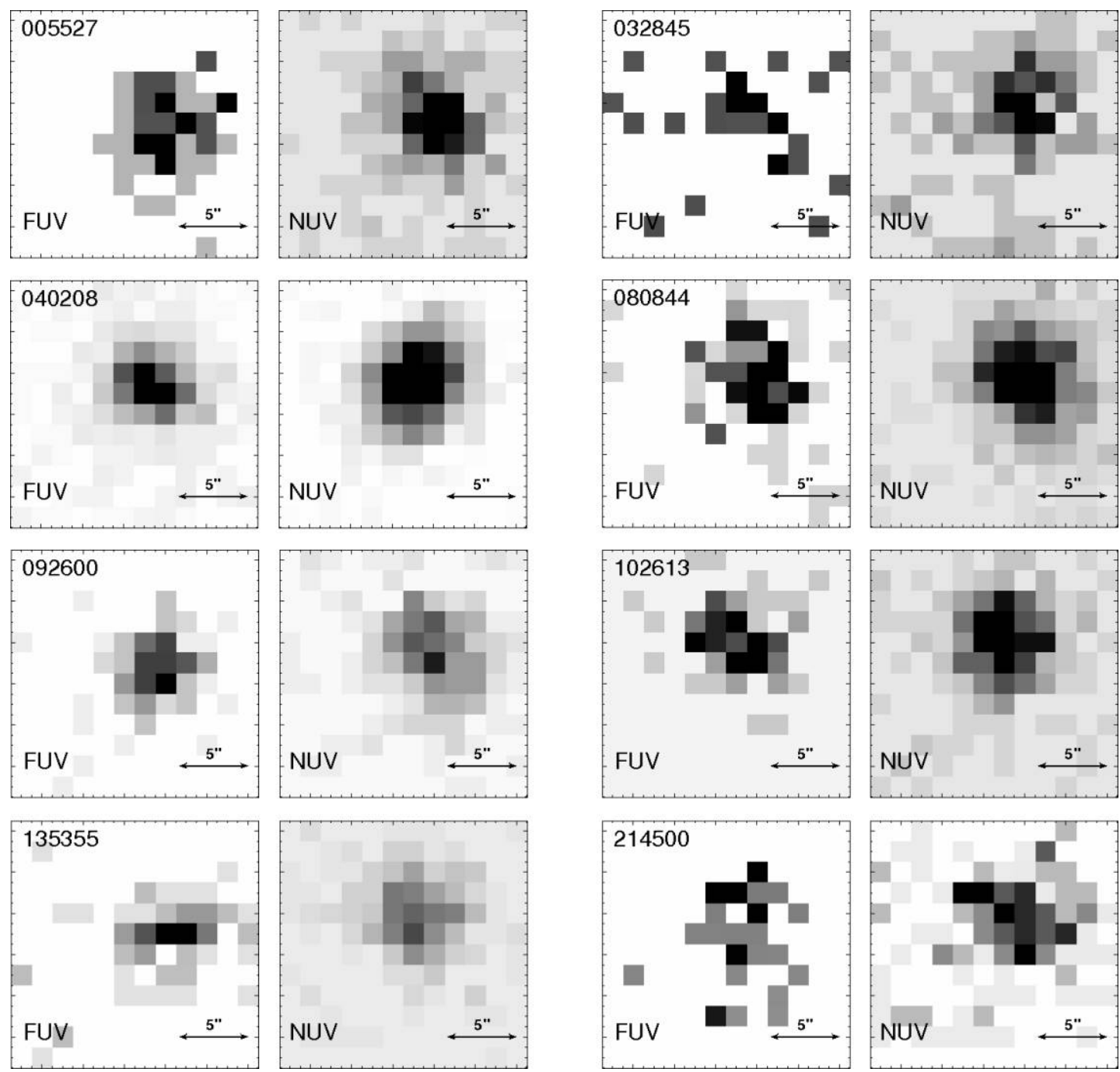

FIG. 1.-GALEX images in the FUVand NUV of the eight objects in our sample. Bars in the lower right of each panel measure $5^{\prime \prime}$ and are comparable to the FWHM of the GALEX PSF. North is up, east is to the left.

axis of the system shows that the two objects are likely to be a counterrotating merger (R. A. Overzier et al. 2008, in preparation).

SDSS J214500.25+011157.6.- This is the only object in our sample for which the ACS image provides a direct match at the wavelength of the GALEX FUV image. The F150LP image shows a very compact but elongated starburst region in which we can discern three different knots. Fainter FUV emission comes from a slightly more extended region of $\sim 1^{\prime \prime}$. The optical morphology in $V_{606}$ is strikingly different. The image is still dominated by the emission surrounding the starburst region, and a faint spiral structure may be present. A companion object appears to have gone straight through the star-forming nucleus as evidenced by its tadpole-like morphology with a faint tail pointing toward the main galaxy and a trail of tidal debris that can be traced back to a location that is about equidistant from the nucleus on the opposite side of the main galaxy.

\subsection{Emission Lines}

Although the sample of UVLGs does not contain any broadline AGNs, we want to make sure that we are studying the morphologies of starburst galaxies rather than those of narrow-line AGNs. The main optical emission line diagnostic diagrams involving the lines $\left[\mathrm{O}\right.$ III] $\lambda 5007, \mathrm{H} \beta,\left[\mathrm{O}_{\mathrm{I}}\right] \lambda 6300, \mathrm{H} \alpha$, [N II] $\lambda 6584$, and the $\left[\mathrm{S}_{\mathrm{II}}\right] \lambda \lambda 6713,6731$ doublet (measured from the SDSS spectra) are shown in Figure 3 (see also H07). The eight objects observed by HST are indicated by the large filled circles and their IDs, while small filled circles indicate objects in our supercompact UVLG sample without HST data. Most of the UVLGs lie along the main star-forming sequence (points) in $\log ([\mathrm{O}$ III $] / \mathrm{H} \beta$ ) versus $\log ([\mathrm{N} \mathrm{II}] / \mathrm{H} \alpha)$ (Fig. 3, left panel), albeit offset toward higher values of $\log ([\mathrm{O} \mathrm{III}] / \mathrm{H} \beta)$ probably due to a more intense ionizing radiation field. It is interesting to note that a similar offset from the main star-forming sequence has been observed for high-redshift LBGs as well (Shapley et al. 2005; Erb et al. 2006a).

About one-quarter of the sample falls in the region between the relations of Kauffmann et al. (2003) (solid line) and Kewley et al. (2006) (dashed line) that is typically populated by objects having a composite spectrum consisting of a metal-rich stellar population and an AGN. When we plot $\log \left(\left[\mathrm{O}_{\mathrm{I}}\right] / \mathrm{H} \alpha\right)$ versus $\log ([\mathrm{O} \mathrm{III}] / \mathrm{H} \beta)$ (middle panel) or $\log \left(\left[\mathrm{S}_{\mathrm{II}}\right] / \mathrm{H} \alpha\right)$ versus $\log \left(\left[\mathrm{O}_{\mathrm{III}}\right] / \mathrm{H} \beta\right)$ (right panel), the objects that were in the AGN/ starburst composite region move to the far, opposite side of the star-forming track (see object SDSS J080844.26+394852.4, which is most relevant to this paper). It is not exactly clear what causes this behavior of the line ratios, but it is consistent with starburst model predictions having very high ionization parameters (Kewley 

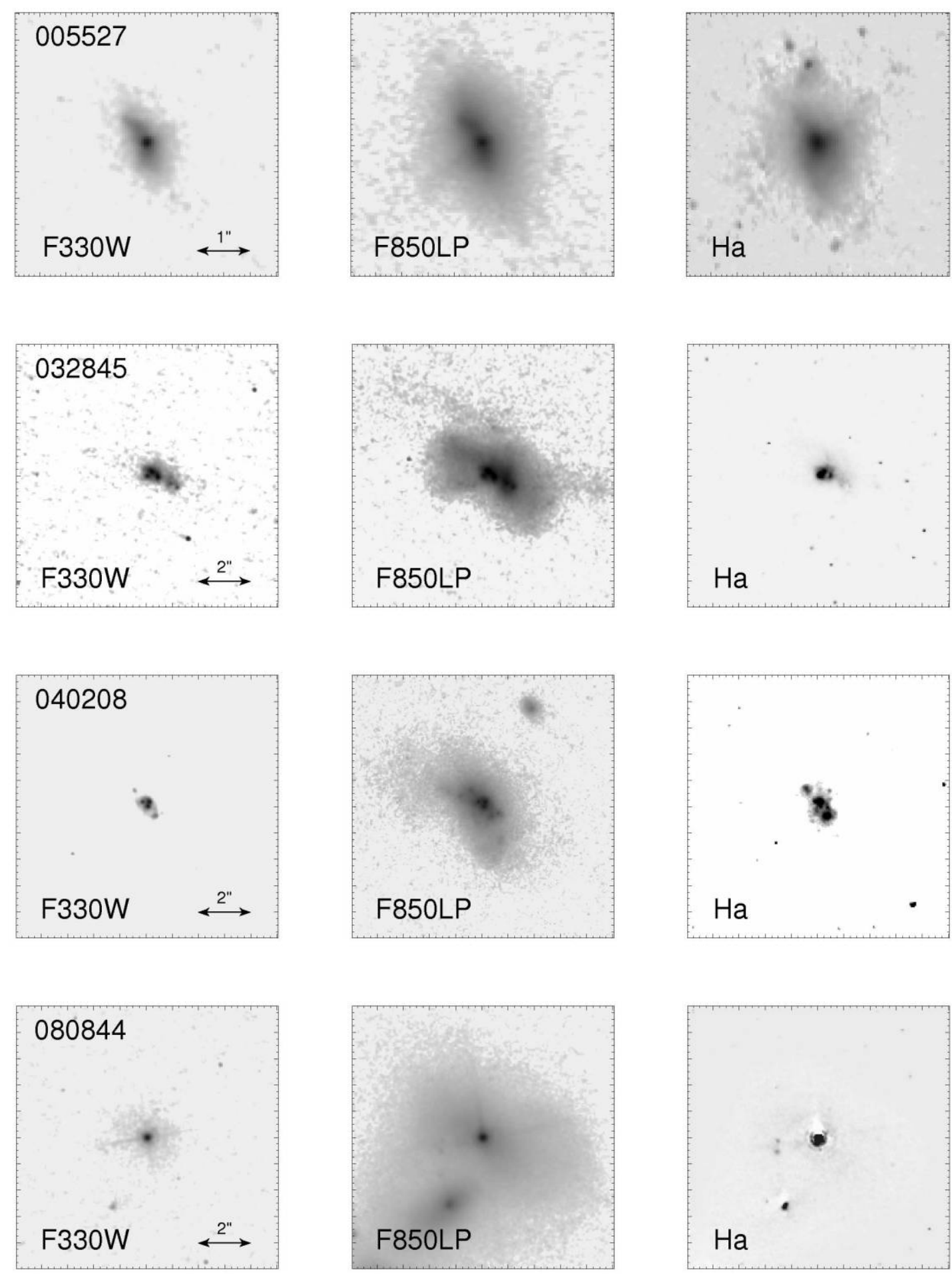

FIG. 2.-HST morphologies in the UV (left), optical (middle), and H $\alpha$ (right). The images were smoothed using a Gaussian filter of 1.5 pixels (FWHM) to reduce some of the noise at the sub-PSF level and shown using a logarithmic stretch. The physical scales range from $1.6 \mathrm{kpc} \mathrm{arcsec}^{-1}$ at $z=0.09$ to $3.2 \mathrm{kpc}$ arcsec ${ }^{-1}$ at $z=0.20$. The F330W image of J092600 and H $\alpha$ image of J214500 are not available. The H $\alpha$ image of J080844 suffers from poor PSF subtraction. North is up, east is to the left.

et al. 2001) as might be expected for our sample. A more detailed analysis of the ionization parameters and the possibly remaining contribution from AGNs will be given elsewhere (R. A. Overzier et al. 2008, in preparation). In any case, Kauffmann et al. (2003) have shown that the continuum emission from local narrow-line AGNs is almost always dominated by its stellar population; this assures us that the HST images accurately reflect the morphology of the stellar light.

\subsection{HST-based Size Measurements}

\subsubsection{UV Sizes}

Our sample of UVLGs is selected to have high surface brightness, which is estimated from the FUV flux and a half-light radius measured from fitting a seeing-convolved exponential profile to the SDSS $u$-band images (see $\S 1, \mathrm{H} 05$, and H07). The resulting seeing-deconvolved sizes were on the order of 1-2 kpc but were 

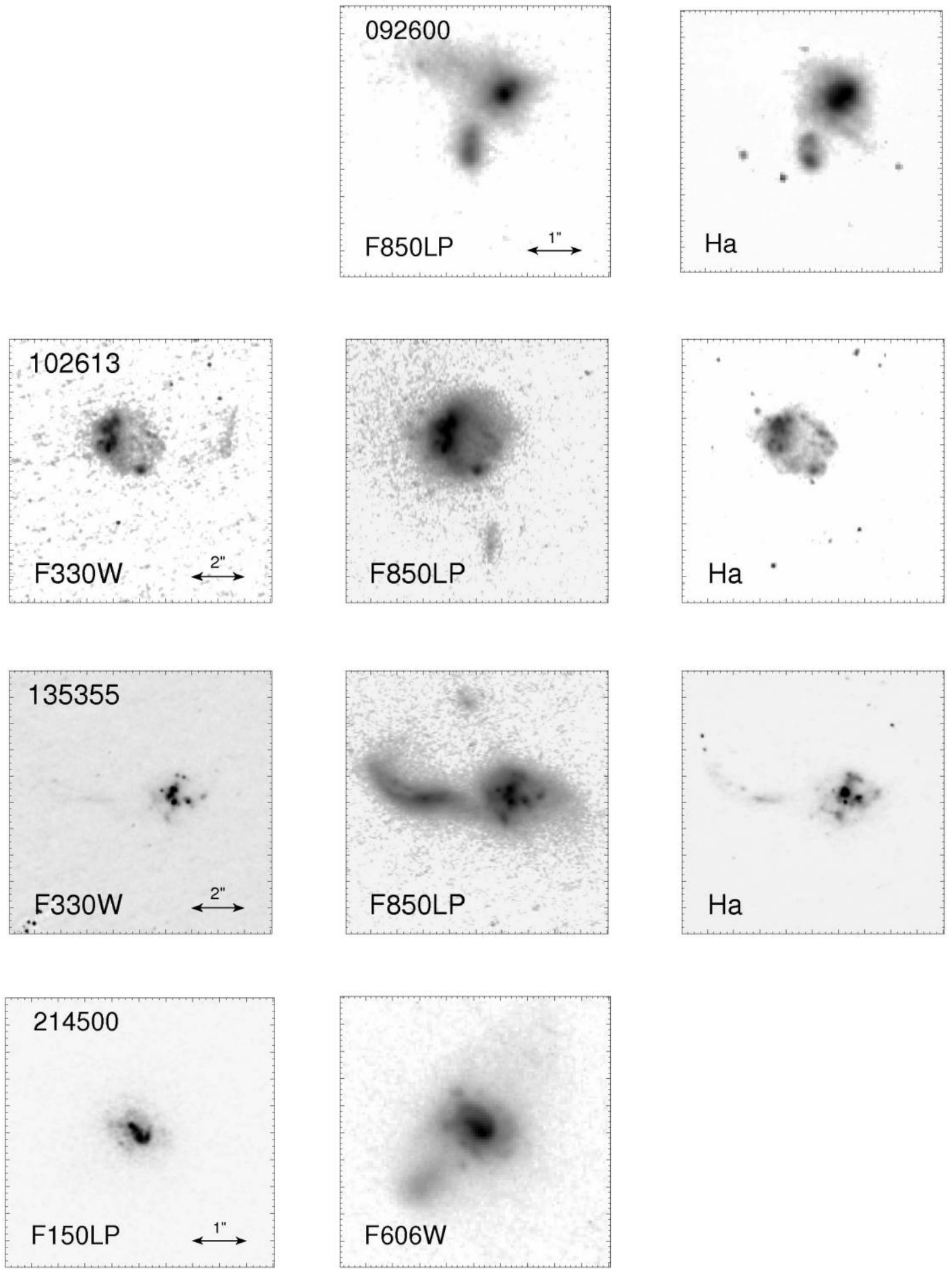

FIG. 2-Continued

somewhat uncertain because most of the UVLGs are only barely resolved in the $u$-band SDSS images. The F330W images are a factor of 10 higher in resolution and thus provide a reliable and independent test for the true sizes of these UVLGs. We have used SExtractor's circular aperture method to estimate the radii; the $50 \%$ and $90 \%$ radii were computed using the light enclosed within 2.5 times the elliptical Kron radius also determined using SExtractor (Table 4). The sizes measured from the HST images are somewhat smaller than our previous estimates based on SDSS. In the case of SDSS J080844, approximately half the light in
F330W is coming from a region that is still unresolved even at the resolution of the HRC $\left(\sim 0.07^{\prime \prime}\right.$, or $\sim 110 \mathrm{pc}$ at $\left.z=0.091\right)$. Excluding this extreme case, the radii range from 0.4 to $1.9 \mathrm{kpc}$.

One could question (see the Appendix) whether the sizes measured in $U_{330}$ or $u$ band (used to calculate the UV surface brightness) are representative for the sizes of the regions that emit in the FUV and NUV as seen by GALEX at the much lower resolution of $\sim 4^{\prime \prime}$ (FWHM). We can test this simply by measuring the slope, $\beta$, of the UV continuum (with $f_{\lambda} \propto \lambda^{\beta}$ ) across the GALEX bands and comparing the flux measured in the HST image to the 

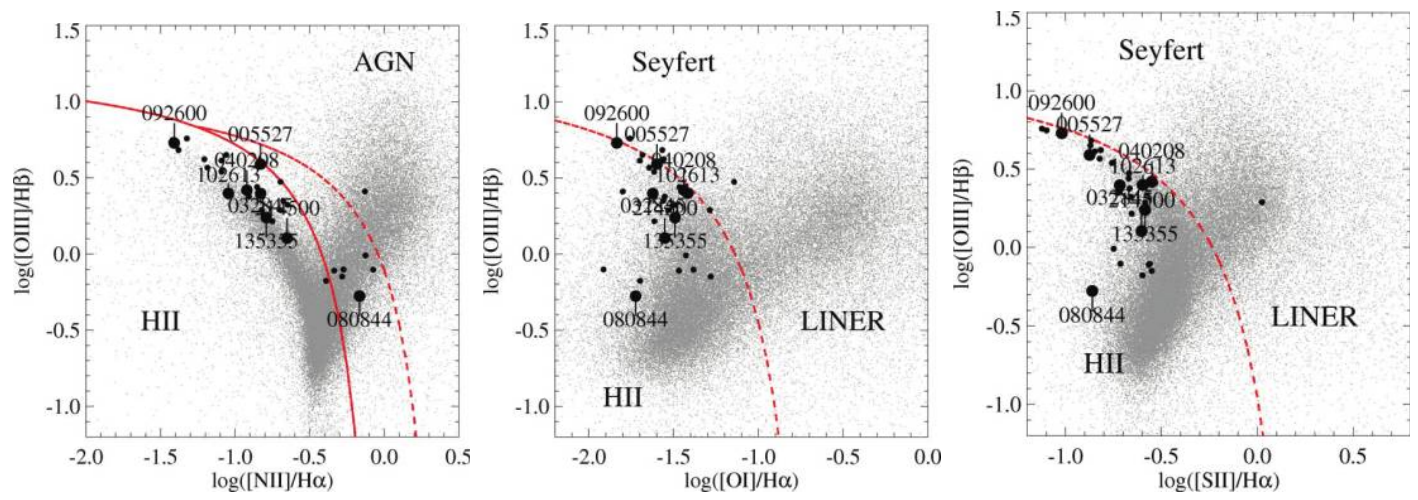

Fig. 3.-Optical emission line diagnostic diagrams $\log ([\mathrm{O}$ III $] / \mathrm{H} \beta)$ vs. $\log \left([\mathrm{N} \mathrm{II]} / \mathrm{H} \alpha)(\right.$ left $), \log \left(\left[\mathrm{O}_{\mathrm{I}}\right] / \mathrm{H} \alpha\right)($ middle $)$, and $\log ([\mathrm{S}$ II $] / \mathrm{H} \alpha)($ right $)$. Points indicate all objects in the GALEX Medium Imaging Survey (MIS) Galex Release 2 (GR2) sample cross matched with the SDSS DR4 sample. The objects that are the subject of the current paper are indicated by the large filled circles. Small filled circles indicate the other objects in our main sample of compact UVLGs that have HST, Spitzer, and radio data either taken or scheduled. The solid line indicates the boundary between regions typically populated by starbursts and Seyferts from Kauffmann et al. (2003). Dashed lines indicate the boundary between objects of starburst spectral type and pure AGNs from Kewley et al. (2006). Objects between the solid and dashed lines in the left panel have typically composite spectra consisting of a metal-rich stellar population and an AGN. Note that, based on these diagnostics, object J080844.26+394852.3 is peculiar. The standard diagnostics diagram (left $)$ indicates it could be of composite type, while it lies well on the starburst side in the other diagrams $($ middle and right). This behavior is typical for galaxies with very high ionization parameters (Kewley et al. 2001). The remainder of our $H S T$ objects lie in the starburst-dominated region, albeit offset toward relatively high values of $\log ([\mathrm{O} \mathrm{mI}] / \mathrm{H} \beta)$. A similar offset has been found for LBGs at high redshift (Shapley et al. 2005; Erb et al. 2006a).

extrapolated value at $U_{330}$. The UV slopes ${ }^{11}$ are given in Table 3, and we note that they are very similar to that of high-redshift LBGs (e.g., Ouchi et al. 2008). The measured flux in $U_{330}$ is lower than the predicted values by a factor of $\sim 1.3$ on average. The measured flux in $u$ band is $\sim 1.2$ times lower than the value extrapolated from the UV. The generally good correspondence between the predicted and measured continuum flux at 1500-3500 $\AA$ indicates that the emission structure seen in the HST and SDSS images is representative of the light distribution in the FUV. A further test is provided by the case of SDSS J214500, for which we have an ACS/SBC image taken through F150LP, a filter that is almost identical to the GALEX FUV filter (see Teplitz et al. 2006). The corresponding FUV surface brightness calculated from the ACS data is $\log _{10} I_{\mathrm{FUV}, \mathrm{ACS}}=9.53 L_{\odot} \mathrm{kpc}^{-2}$, in agreement with $\mathrm{H} 07$.

\subsection{2. $\mathrm{H} \alpha$ and Optical Sizes}

The distribution of the optical and $\mathrm{H} \alpha$ light need not be the same. We find, however, that the sources are compact at all observed wavelengths (Table 4) and the starbursts dominate the structures. The half-light radii measured from the continuum-

\footnotetext{
${ }^{11}$ Calculated from $\beta_{\text {GALEX }}=2.32\left(m_{\mathrm{FUV}}-m_{\mathrm{NUV}}\right)_{\mathrm{AB}}-2$.
}

subtracted $\mathrm{H} \alpha$ images are slightly larger than those measured from $U_{330}(\sim 0.5-2 \mathrm{kpc})$. In some cases, the $\mathrm{H} \alpha$ morphology suggests an outflow (SDSS J005527, and perhaps J032845 and J092600; see Fig. 2), but this does not affect the measured sizes. The half-light radii in F850LP are typically $1-2 \mathrm{kpc}$, with the exception of SDSS J135355, which has $r_{50,850} \approx 4 \mathrm{kpc}$ (note, however, that it has a nearby companion). Both the $50 \%$ and $90 \%$ radii are typically twice as large as the UV size $(\sim 2-5 \mathrm{kpc})$, indicating that these systems have relatively faint, underlying structures that were already in place prior to the current episode of star formation.

\subsection{UV-Optical Colors}

We have used SExtractor to derive (circular) $U_{330}-z_{850}$ radial color profiles out to the $90 \%$ flux radius. The results are shown in Figure 4. Most objects have large gradients in their UV-optical color profile, with objects typically being bluer near the center and redder farther out. Because the profiles were calculated with respect to the centroid of the objects in the optical image, for some objects (J032845, J040208, and J102613) the bluest regions are sometimes slightly offset from the nominal center of the galaxy (see Fig. 2). We have defined an "inner" color measured within the $U_{330}$ half-light radius and an "outer" color measured over the

TABLE 4

Size Measurements and Colors

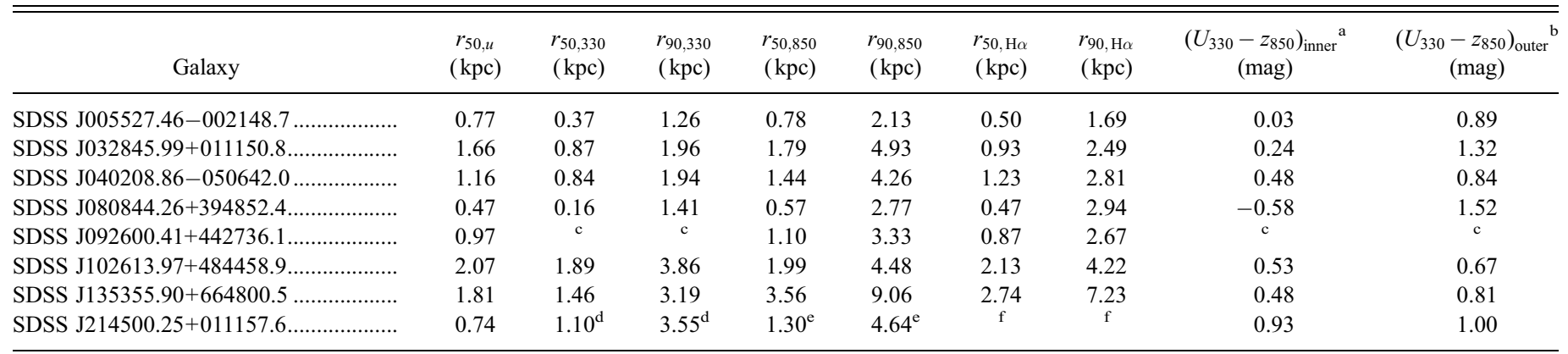

\footnotetext{
${ }^{a}$ Inner color measured within $r_{50,330}$ (not corrected for internal reddening).

${ }^{\mathrm{b}}$ Outer color measured between $r_{50,850}$ and $r_{90,850}$ (not corrected for internal reddening).

c We did not obtain data in F330W.

d These measurements correspond to the ACS/SBC F150LP data, instead of ACS/HRC F330W.

e These measurements correspond to the WFPC2 F606W data, instead of ACS/WFC F850LP.

${ }^{\mathrm{f}}$ We did not obtain data in $\mathrm{H} \alpha$.
} 


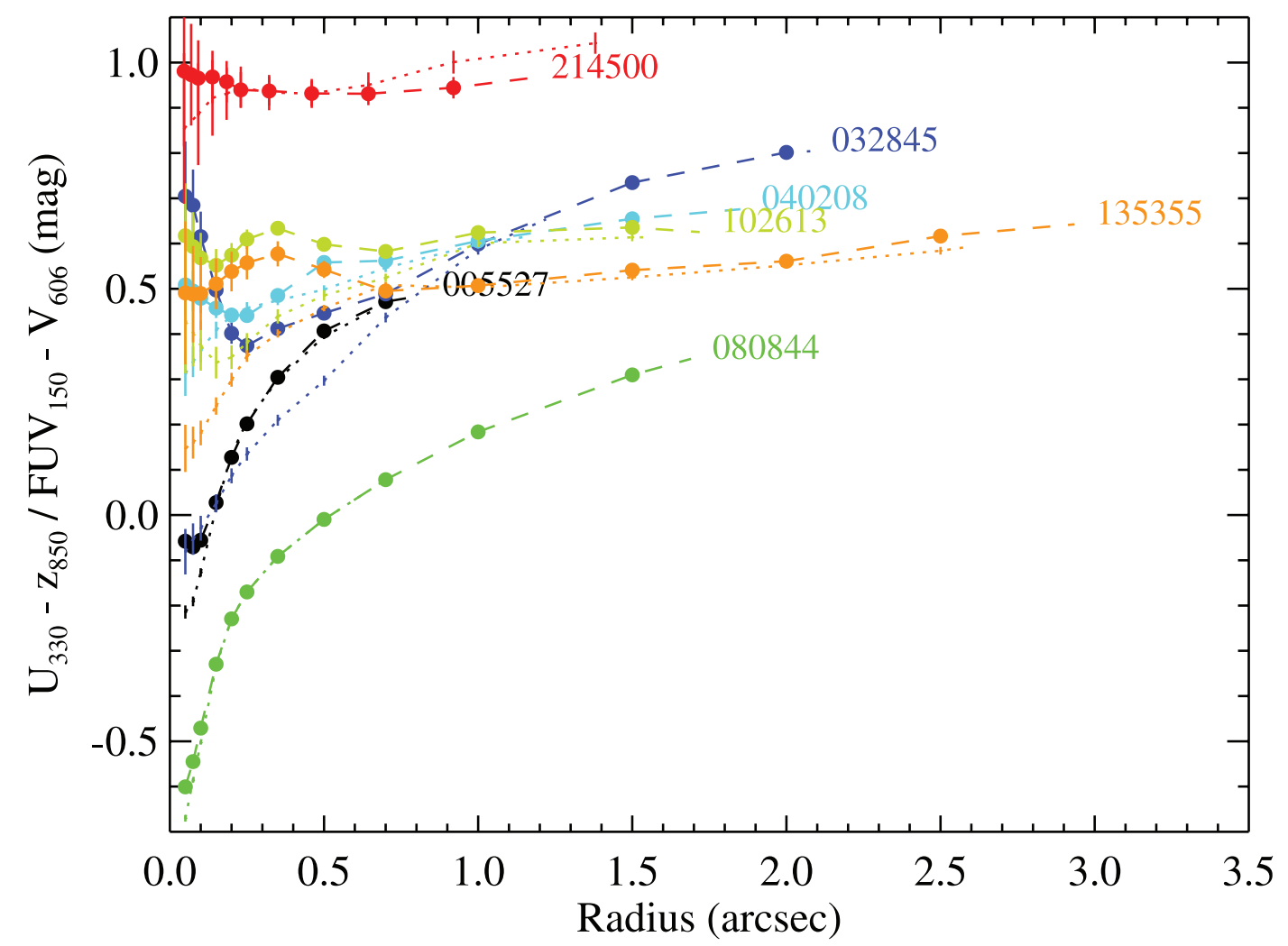

FIG. 4.-UV-optical radial color profiles of the UVLGs shown in Fig. 2. Lines indicate the (cumulative) color determined in circular apertures measured from the centroids of the object detected in the optical image out to the $90 \%$ flux radius (dashed colored lines) and with respect to the object centroids defined in the UV image (dotted colored lines). The UVLGs are generally very blue within approximately the half-light radius and become redder at larger radii. The objects often have steeper inner color gradients when the UV image is used for object detection (dotted lines), compared to when the optical image is used (dashed lines) due to the different positions of the object centroids in the UV and optical. For J214500, the UV-optical color corresponds to FUV $\mathrm{V}_{150}-V_{606}$, whereas for the other objects the colors measured are $U_{330}-z_{850}$. Errors are $3 \sigma$.

region between the $50 \%$ and $90 \%$ radii in $z_{850}$ (see Table 4 ). In all cases the inner color is bluer than the outer color, and the steepest color gradients are seen for the most compact objects (in $U_{330}$ ). This indicates that these objects are composites of recent, central starbursts within older (or more dusty) extended structures.

\subsection{Extinction}

For starburst galaxies there exists a good correlation between the amount of UV flux that is absorbed by dust (usually taken to be a foreground "screen") and the amount of flux (re)emitted in the far-IR (Meurer et al. 1997; Calzetti 2001; Kong et al. 2004; Seibert et al. 2005). To estimate the internal extinction for our sample, we have calculated the attenuation in the FUV, $A_{\mathrm{FUV}}$, using the ratio of the bolometric dust luminosity, $L_{\mathrm{TIR}}$, to bolometric FUV luminosity, $L_{\mathrm{FUV}}$, and the fitting formula of Burgarella et al. (2005):

$$
A_{\mathrm{FUV}}=a_{1} x^{3}+a_{2} x^{2}+a_{3} x+a_{4}
$$

with $\left(a_{1}, a_{2}, a_{3}, a_{4}\right)=(-0.028,0.392,1.094,0.546)$ and $x=$ $\log _{10}\left(L_{\mathrm{TIR}} / L_{\mathrm{FUV}}\right)$. The bolometric dust luminosity is given by (Dale \& Helou 2002)

$$
\mathcal{L}_{\mathrm{TIR}}=\xi_{1} \nu_{24} L_{24}+\xi_{2} \nu_{70} L_{70}+\xi_{3} \nu_{160} L_{160}
$$

with $\left(\xi_{1}, \xi_{2}, \xi_{3}\right)=(1.559,0.7686,1.347)$. Although we do not have observed flux densities at $160 \mu \mathrm{m}$, we can estimate $160 \mu \mathrm{m}$ using the strong empirical correlation between $\left(f_{8} / f_{24}\right)_{\text {dust }}$ and $\left(f_{70} / f_{160}\right)$ by employing the synthetic models of Dale \& Helou (2002). The correlation arises because more intense radiation fields have larger $\left(f_{70} / f_{160}\right)$ due to hotter large grains and smaller $\left(f_{8} / f_{24}\right)_{\text {dust }}$ due to larger emission by small grains at $24 \mu \mathrm{m}$. The dust emission at 8.0 and $24 \mu \mathrm{m}$ was calculated from the observed flux densities using $\left(f_{8}\right)_{\text {dust }}=f_{8}-0.232 f_{3.6}$ and $\left(f_{24}\right)_{\text {dust }}=f_{24}-$ $0.032 f_{3.6}$, which includes a correction for the contribution due to stars based on the $3.6 \mu \mathrm{m}$ flux (Dale et al. 2005). Finally, we calculate the extinction using the formula $E(B-V)_{\text {stars }}=A_{\mathrm{FUV}} / k(\lambda)$ with $k(\lambda) \approx 10.77$ (Calzetti 2001). The extinction ranges from $E(B-V) \approx 0.01$ to 0.14 . The IR flux densities, bolometric luminosities, $A_{\mathrm{FUV}}$, and extinction are given in Table 5 . The extinction is needed in $\S 4$ when we estimate masses and ages from the data using a set of model star formation histories. We refer the reader to Basu-Zych et al. (2007) for a more detailed discussion and analysis of the extinction properties of this sample using alternative estimators.

\subsection{Morphologies}

\subsubsection{Gini Coefficient, $M_{20}$, and Concentration}

In order to compare the morphologies of the UVLGs to those of nearby galaxies and of LBGs at high redshift, we calculated the Gini coefficient ( $G$; a measure of the equality with which the flux is distributed across a galaxy), $M_{20}$ (the $\log$ of the ratio of the second-order moment of the pixels containing the $20 \%$ brightest flux to the total second-order moment), and concentration (C; 5 times the log of the ratio of the circular radii containing $80 \%$ and $20 \%$ of the flux). To calculate the structural parameters, we follow the procedures described in full detail in Lotz et al. $(2004,2006)$. First, we use SExtractor to make an object segmentation map and mask out neighboring objects. The image is 
TABLE 5

INFRAREd Photometry and Extinction

\begin{tabular}{|c|c|c|c|c|c|c|c|c|c|}
\hline Galaxy & $\begin{array}{c}f_{3.6} \\
(\mu \mathrm{Jy})\end{array}$ & $\begin{array}{c}f_{8} \\
(\mu \mathrm{Jy})\end{array}$ & $\begin{array}{c}f_{24} \\
(\mathrm{mJy})\end{array}$ & $\begin{array}{c}f_{70} \\
(\mathrm{mJy})\end{array}$ & $\begin{array}{l}f_{160}{ }^{\mathrm{a}} \\
(\mathrm{mJy})\end{array}$ & $\begin{array}{c}L_{\mathrm{TIR}}{ }^{\mathrm{b}} \\
\left(10^{10} L_{\odot}\right)\end{array}$ & $\begin{array}{c}L_{\mathrm{FUV}}{ }^{\mathrm{c}} \\
\left(10^{10} L_{\odot}\right)\end{array}$ & $\begin{array}{l}A_{\mathrm{FUV}}{ }^{\mathrm{d}} \\
(\mathrm{mag})\end{array}$ & $\begin{array}{l}E(B-V)_{\mathrm{int}}^{\mathrm{e}} \\
\quad(\mathrm{mag})\end{array}$ \\
\hline SDSS J005527.46-002148.7 …….......... & $725.8 \pm 33.2$ & $4280.5 \pm 80.8$ & $33.2 \pm 0.4$ & $163.4 \pm 6.4$ & 115.4 & 16 & 3.2 & 1.48 & 0.14 \\
\hline SDSS J040208.86-050642.0 .................. & $131.6 \pm 34.8$ & $415.3 \pm 66.6$ & $1.1 \pm 0.1$ & $<19.8^{\mathrm{f}}$ & 25.5 & $<1.6$ & 2.8 & 0.31 & 0.03 \\
\hline SDSS J080844.26+394852.4.................. & $1048.6 \pm 39.8$ & $5786.5 \pm 66.2$ & $39.9 \pm 0.6$ & $156.0 \pm 6.8$ & 117.0 & 4.1 & 2.0 & 0.91 & 0.08 \\
\hline SDSS J092600.41+442736.1................. & $90.7 \pm 4.2$ & $229.4 \pm 24.0$ & $3.4 \pm 0.4$ & $20.7 \pm 3.7$ & 10.2 & 2.1 & 5.5 & 0.16 & 0.01 \\
\hline SDSS J102613.97+484458.9................. & $176.6 \pm 36.2$ & $614.5 \pm 56.8$ & $4.2 \pm 0.3$ & $42.5 \pm 4.2$ & 31.6 & 3.7 & 2.7 & 0.70 & 0.06 \\
\hline SDSS J214500.25+011157.6 $\ldots \ldots \ldots \ldots \ldots \ldots$ & $200.4 \pm 31.5$ & $1617.5 \pm 64.2$ & $5.0 \pm 0.4$ & $23.0 \pm 5.5$ & 27.7 & 4.2 & 2.6 & 0.79 & 0.07 \\
\hline
\end{tabular}

${ }^{\text {a }}$ Estimated using the observed flux densities at 3.6, 8.0, 24, and $70 \mu \mathrm{m}$ and the dust models of Dale \& Helou (2002).

b Bolometric dust luminosity.

${ }^{c}$ Bolometric FUV luminosity.

d FUV attenuation calculated from $L_{\mathrm{TIR}} / L_{\mathrm{FUV}}$ and using the fitting formula of Burgarella et al. (2005).

${ }^{\mathrm{e}}$ Internal (stellar) extinction $E(B-V)_{\mathrm{int}}=A_{\mathrm{FUV}} / k(\lambda)($ Calzetti 2001).

${ }^{\mathrm{f}}$ Indicates a $3 \sigma$ upper limit.

background subtracted, and we calculate an initial Petrosian radius ( $r_{\mathrm{P}}$ with $\left.\eta \equiv 0.2\right)$ using the object center and (elliptical) shape information from SExtractor. We then smooth the image by $\sigma=r_{\mathrm{P}} / 5$ and create a new segmentation map by selecting those pixels that have a surface brightness higher than the mean surface brightness at the Petrosian radius. We recalculate the object center by minimizing the second-order moment of the flux and then recalculate the Petrosian radius in the original image using this center. The total flux is defined as the flux within a radius of $1.5 r_{\mathrm{P}}$, and $C$ is then calculated in circular apertures. The individual measurements are listed in Table 6 , and the results are shown in Figure 5. For each object, the $U_{330}$ measurements are plotted as stars and the $z_{850}$ as circles and are connected by a dotted line. For J080844, $G$ - and $C$-values in $U_{330}$ must be considered lower limits, and its $M_{20}$ value is an upper limit because the central pixel of the unresolved component contains $\approx 20 \%$ of the total flux.

Although our sample is small, we attempt to make a general comparison with the morphologies of nearby galaxies. As shown in Figure 5, the UVLGs populate the region roughly defined by $M_{20} \lesssim-1.3$ and $0.53 \lesssim G \lesssim 0.7$ (left panel) and $3 \lesssim C \lesssim 5$ (right panel). We can compare these measurements to the various divisions of morphological parameter space discussed by Lotz et al. (2006). Our UVLGs have smaller $M_{20}$ than merging galaxies with two clearly separated nuclei $\left(M_{20} \gtrsim-1.1\right.$; left hatched region), larger $G$ than the expectations for exponential disks (dotted box), $M_{20}$ similar or larger than those expected for bulge-dominated objects (right hatched region), and $G$ and $C$ that are either similar to or smaller than those expected for bulge-dominated galaxies seen face-on (solid box). We do not find any systematic differences between the morphologies measured in $U_{330}$ and $z_{850}$ for the sample as a whole. The morphological parameters in the UV and optical are thus dominated by the same (bright) regions, even though some of the objects possess very faint, extended structures in $z_{850}$.

Figure 5 shows that two UVLGs qualify as bulge-dominated in $U_{330}$, while four objects qualify as bulge-dominated in $z_{850}$. None of the objects qualify as a pronounced merger, and only one object lies just on the border of the exponential disk region in $z_{850}$, with the remainder populating the regions in between. The overall distribution of morphologies is very similar to that of star-forming galaxies at $z \sim 1.5$ and LBGs at $z \sim 4$ selected from GOODS and the UDF. Lotz et al. (2006) find median values of $G \sim 0.55, M_{20} \sim$ -1.5 , and $C \sim 3.3$ at $z \sim 1.5$ and $G \sim 0.58, M_{20} \sim-1.6$, and $C \sim 3.8$ at $z \sim 4$ (Fig. 5, encircled plus signs). However, a fair comparison of the morphologies of UVLGs with the higher redshift comparison samples should be carried out at the same signalto-noise ratio $(\mathrm{S} / \mathrm{N})$ and resolution. In the next section we carry out such a comparison.

\subsubsection{Redshift Simulations}

We now investigate whether the conclusions of the previous subsection are still upheld if the analysis is carried out using images that are simulated to have the same depth and resolution as LBGs observed at high redshift.

TABLE 6

Morphological Parameters

\begin{tabular}{|c|c|c|c|c|c|c|c|c|}
\hline Galaxy & $\begin{array}{c}r_{\mathrm{P}, 330} \\
(\operatorname{arcsec})\end{array}$ & $G_{330}$ & $M_{20,330}$ & $C_{330}$ & $\begin{array}{c}r_{\mathrm{P}, 850} \\
(\operatorname{arcsec})\end{array}$ & $G_{850}$ & $M_{20,850}$ & $C_{850}$ \\
\hline SDSS J005527.46-002148.7 ……………..... & 0.55 & 0.63 & -2.41 & 4.73 & 0.83 & 0.58 & -1.98 & 3.53 \\
\hline SDSS J032845.99+011150.8 & 1.66 & 0.66 & -1.48 & 3.97 & 2.62 & 0.58 & -1.55 & 3.34 \\
\hline SDSS J040208.86-050642.0 …………....... & 1.02 & 0.56 & -1.60 & 3.29 & 1.92 & 0.60 & -1.98 & 3.92 \\
\hline 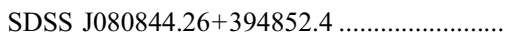 & 0.13 & $>0.57$ & $<-2.55$ & $>3.01$ & 2.97 & 0.66 & -3.23 & 7.11 \\
\hline 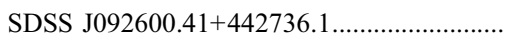 & a & a & a & a & 1.29 & 0.73 & -1.60 & 3.27 \\
\hline SDSS J102613.97+484458.9.............................. & 2.21 & 0.60 & -1.31 & 3.28 & 1.92 & 0.58 & -1.48 & 2.93 \\
\hline SDSS J135355.90+664800.5 …….................... & 1.26 & 0.60 & -1.27 & 2.88 & 1.29 & 0.53 & -1.57 & 2.88 \\
\hline 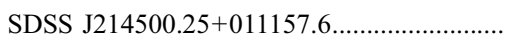 & $0.75^{\mathrm{b}}$ & $0.56^{\mathrm{b}}$ & $-1.47^{\mathrm{b}}$ & $2.95^{\mathrm{b}}$ & $1.40^{\mathrm{c}}$ & $0.61^{\mathrm{c}}$ & $-1.80^{\mathrm{c}}$ & $3.56^{\mathrm{C}}$ \\
\hline
\end{tabular}

\footnotetext{
${ }^{\mathrm{a}}$ We did not obtain data in $\mathrm{F} 330 \mathrm{~W}$.

b These measurements correspond to the ACS/SBC F150LP data, instead of ACS/HRC F330W.

c These measurements correspond to the WFPC2 F606W data, instead of ACS/WFC F850LP.
} 

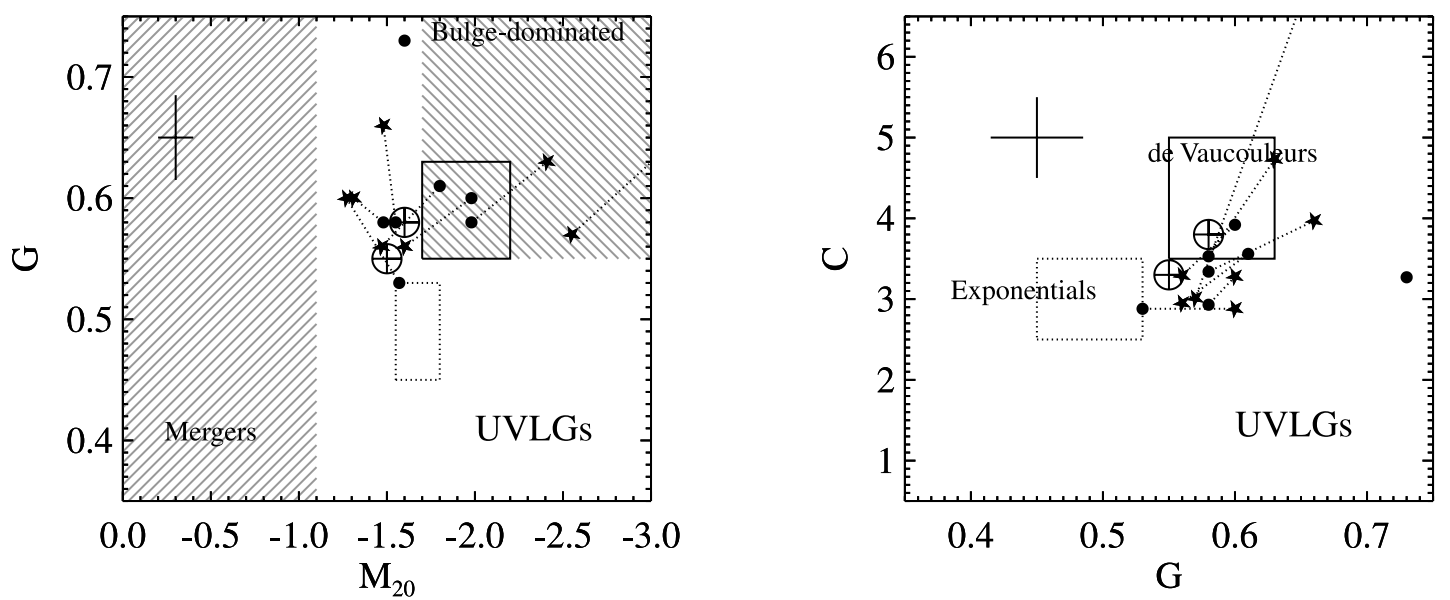

FIG. 5.-Distribution of morphological parameters $M_{20}$ vs. $G($ left $)$ and $G$ vs. $C$ (right $)$ for the sample of $z \sim 0.15$ UVLGs. Morphologies measured from the UV images are indicated by stars, morphologies measured from the optical images by filled circles. Dotted lines connect the UV and optical morphologies measured for each source. For comparison, we have indicated the following (all adapted from Lotz et al. 2006): regions populated by likely mergers having multiple nuclei (left hatched area) and by bulge-dominated objects (right hatched area), regions occupied by simulated de Vaucouleurs and exponential profiles (top and bottom boxes, respectively), the median values found for star-forming galaxies at $z \sim 1.5$ and LBGs at $z \sim 4$ in GOODS (encircled plus signs), and the typical errors for the high-redshift samples (large plus signs).

We follow common practice (see, e.g., Giavalisco et al. 1996a; Hibbard \& Vacca 1997; Bouwens et al. 1998; Papovich et al. 2003; Conselice 2003; Lotz et al. 2004) and apply corrections for cosmological surface brightness dimming and for changes in physical resolutions to the $U_{330}$ images, in order to match the instrumental conditions under which our objects would be observed in typical surveys when placed at $z=1-4$.

The first step of the procedure is to rebin the $U_{330}$ images by a factor $b=\left(\theta_{1} / \theta_{2}\right)\left(s_{2} / s_{1}\right)$, where $\theta_{i}$ is the angle on the sky of an object of fixed size $d$ at $z=z_{i}$ and $s_{i}$ is the instrumental pixel scale (in arcsec pixel ${ }^{-1}$ ). The rebinning factor can be expressed in terms of either the angular diameter distance, $D_{A_{i}}=d / \theta_{i}$, or the luminosity distance, $D_{L_{i}}=\left(1+z_{i}\right)^{2} D_{A_{i}}$, at low and high redshift $\left(z_{2}>z_{1}\right)$ :

$$
b=\frac{D_{A_{2}}}{D_{A_{1}}} \frac{s_{2}}{s_{1}}=\left(\frac{1+z_{1}}{1+z_{2}}\right)^{2} \frac{D_{L_{2}}}{D_{L_{1}}} \frac{s_{2}}{s_{1}} .
$$

The second step is to reduce the surface brightness of each (rebinned) pixel according to the relative amount of cosmological dimming of a galaxy at $z_{2}$ with respect to that at $z_{1}$. We calculate the scaling by making use of the fact that the absolute rest-frame magnitude (or luminosity) of the object before and after redshifting will be conserved $\left[M_{\lambda_{2} /\left(1+z_{2}\right)}=M_{\lambda_{1} /\left(1+z_{1}\right)}\right.$ with matched filters so that $\left.\lambda_{2}=\lambda_{1}\left(1+z_{2}\right) /\left(1+z_{1}\right)\right]$.

In order to compare our results to the high-redshift samples of Lotz et al. (2006), objects were simulated at $z=1.5$ in $B_{435}$ and at $z=3.0$ and 4.0 in both $V_{606}$ and $i_{775}$. Objects were simulated at the depths of GOODS $\left(3,2.5\right.$, and 2.5 orbits in $B_{435}, V_{606}$, and $i_{775}$, respectively) and the UDF (56, 56, and 150 orbits in $B_{435}$, $V_{606}$, and $i_{775}$, respectively). For completeness and future reference, we also simulated the sample at the shallower COSMOS survey in the $I_{814}$ filter (one orbit). For each filter and for each redshift, we used the HST images that correspond most closely in terms of rest-frame central wavelength. Where necessary, we rescaled the flux of the input images assuming the UV slopes determined in $\S 3.5$. The final steps of the simulation consist of applying Poissonian noise to the simulated profiles based on the typical exposure times of COSMOS/GOODS/UDF, convolving the images with a Gaussian to match the desired output point-spread function (PSF) size $\left(\sim 0.12^{\prime \prime}\right)$, and placing the simulated object inside an empty region in a COSMOS, GOODS, or UDF image to obtain a realistic background. The simulated pixel scale was $0.03^{\prime \prime}$ pixel $^{-1}$ for GOODS and the UDF and $0.05^{\prime \prime} \mathrm{pixel}^{-1}$ for COSMOS.

The rest-frame UV images of Haro 11 and VV 114 (see Fig. 6, top panels) were artificially redshifted in an identical manner using a two-step process. First, we simulated their F330W images at a redshift $(z=0.15)$, depth $(2500 \mathrm{~s})$, plate scale $\left(0.025^{\prime \prime}\right.$ pixel $\left.^{-1}\right)$, and seeing $\left(\sim 0.075^{\prime \prime}\right)$ comparable to our observations of the UVLGs. These images are shown in the bottom panels of Figure 6. Both Haro 11 and VV 114 possess multiple bright nuclei $\left(\sim 1^{\prime \prime}\right.$ apart) when observed in $U_{330}$ at $z=0.15$. VV 114 also shows a significant amount of diffuse emission in between the
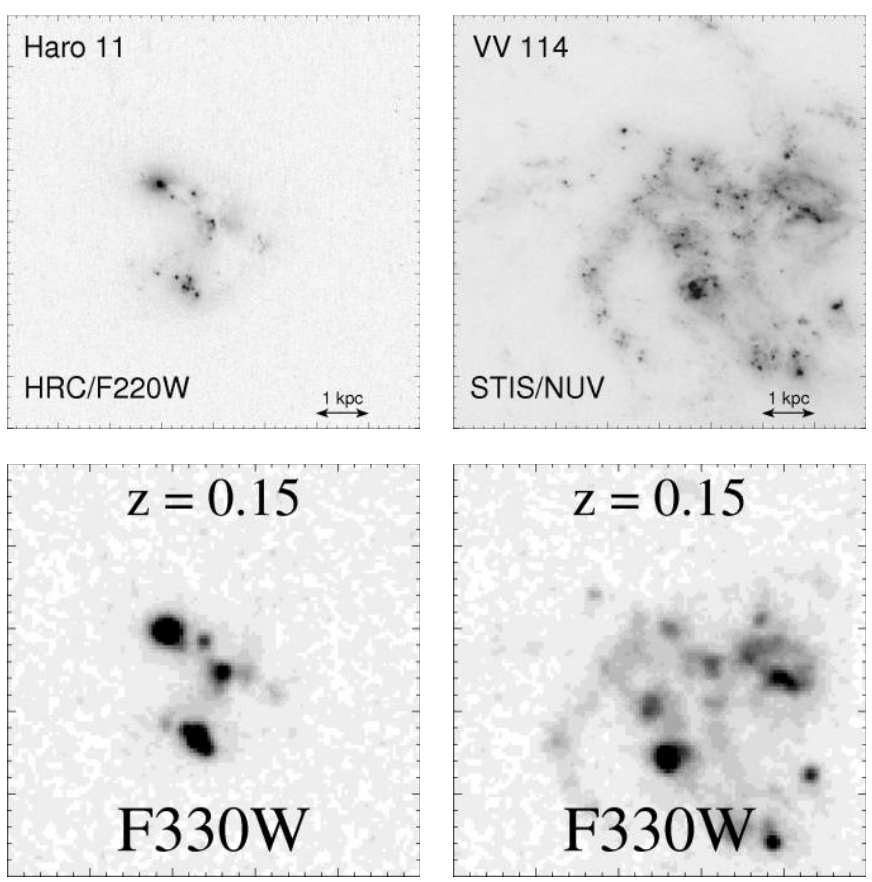

FIG. 6.-Top: NUV archival images of the local starburst galaxies Haro 11 and VV 114, both at $z=0.02$. The image of Haro 11 was taken with the ACS/HRC (Program 10575; PI: G. Östlin). The image of VV 114 was taken with STIS (Program 8201; PI: G. Meurer). See $\S 2.4 .1$ for details. Bottom: Simulated F330W images of Haro 11 (left) and VV 114 (right) at $z=0.15$ for comparison with our UVLG sample. The images measure $3^{\prime \prime} \times 3^{\prime \prime}$. See $\S 3.6 .2$ for details. 
knots. Next, these images were used to simulate how these objects would appear at high redshift, analogous to the UVLG simulations described above. To match the desired rest-frame wavelengths of the output filters, small flux extrapolations were performed using $\beta=-1.4$ for both objects as measured by Goldader et al. (2002) and Bergvall et al. (2006).

\subsubsection{Results}

Figures 7, 8, and 9 compare postage stamps of the local LBG analogs (including Haro 11 and VV 114) that are simulated at the depths of the GOODS, UDF, and COSMOS surveys. Results are shown at the three different redshifts discussed above $(z=1.5$, 3.0, and 4.0). The images demonstrate that as redshift increases, components that are well separated at low redshift blend due to the lower spatial resolution, and that low surface brightness features are lost due to surface brightness dimming. The latter effect is most severe in the shallow COSMOS data and least severe in the deep UDF data.

Figure 10 shows how the measured morphological parameters $G$ (top panel), $M_{20}$ (middle panel), and $C$ (bottom panel) change as a function of redshift and survey depth. There is a systematic drop of $\sim 0.05-0.10$ in $G$ from $z \sim 0.15$ to $\sim 1.5$, followed by a further less significant decrease of a few hundredths out to $z=4$. The first decrease is caused by the strong drop in resolution, as well as the loss of faint extended features that cause the flux to be more evenly distributed over the galaxy profile. Simulations by Lotz et al. (2006) show that the second decrease is mainly caused by the loss of low surface brightness pixels lowering $G$ somewhat further at $z>1.5$. The loss of low surface brightness features with redshift also tends to increase $M_{20}$ (the total second-order moment is lowered, while the second-order moment of the $20 \%$ brightest flux stays roughly constant) and tends to lower the concentration because the $80 \%$ flux radii are systematically underestimated. The measurements at the COSMOS depth show quite a large scatter with respect to GOODS and the UDF, indicating that the lower $\mathrm{S} / \mathrm{N}$ and its larger pixel scale result in relatively unstable morphology measurements for LBGs.

The main results of our morphological comparison are presented in Figure 11. We compare the UVLG morphologies ( filled stars) with those of the low-redshift LBG analogs (marked "V" and " $\mathrm{H}$ ") and the high-redshift comparison sample ( plus signs) of Lotz et al. (2006). In order to make sure that the morphologies are measured in a consistent manner, we used our own code to recalculate the morphologies of the $55 z \sim 1.5$ starburst galaxies in GOODS (bottom panels) and the $82 z \sim 4$ LBGs in GOODS (middle panels) and the UDF (top panels) analyzed by Lotz et al. (2006). Lotz et al. (2006) showed that the morphologies of the $z \sim 1.5$ sample are very similar to that of the $z \sim 4$ sample. The distribution of the UVLGs is very similar to that of the high-redshift objects as well, although the latter has a larger scatter due to the much larger sample size.

\subsubsection{Comparison with Haro 11 and VV 114}

Haro 11 and VV 114 (Fig. 11, marked "V" and "H") have higher $M_{20}$ and lower $G$ and $C$ when compared to the UVLGs. We can understand this given that both objects possess several bright nuclei with a separation that enables them to be significantly resolved even when simulated at $z=0.15-4.0$. Their qualitative morphology perhaps most closely resembles that of the high-redshift "clump cluster galaxies" (see, e.g., Elmegreen et al. 2004). Although objects with similar morphological characteristics are certainly present in high-redshift samples, they do not make up the majority of LBGs as can be seen from the distribution in morphological types of LBGs (e.g., Lotz et al. 2006;
Ravindranath et al. 2006; Elmegreen et al. 2007; see also Fig. 11 in this paper).

It is important to note that our GALEX/SDSS selection of local LBG analogs at $z \approx 0.1-0.3$ is likely biased against finding objects having such widely separated nuclei as Haro 11 and VV 114. Because we selected objects principally on having a high FUV luminosity, as well as a high UV surface brightness based on the half-light radius measured in the $u$ band, we are most sensitive to objects that are not or barely resolved in SDSS. The surface brightness requirement is necessary in order to obtain a relatively clean separation between the LBG analogs on one hand and large, UV-luminous (predominantly spiral) galaxies on the other. However, we will further investigate whether we can find more LBG analogs of the type of Haro 11/VV 114 by looking closer at the objects that straddle the boundary of the LBG analog selection criteria in a future paper (R. A. Overzier et al. 2008, in preparation).

\subsubsection{Summary}

We conclude that the morphologies of the UVLGs cannot be distinguished from the morphologies of high-redshift LBGs when measured from the redshifted images simulated at the same depth as GOODS and the UDF. This further strengthens our conclusion that the relatively nearby supercompact UVLGs and high-redshift LBGs are very similar.

In the following sections, we turn our attention again to the undegraded HST images of Figure 2. In $\S 4$ we study in detail the nature of the starburst regions, and in $\S 5$ we discuss the connection between morphology and the mechanisms responsible for triggering the vigorous star formation observed in these local LBG analogs.

\section{SUPER STARBURST REGIONS}

As discussed in $\S 3$, in all our sources the UV light is distributed in a series of bright, unresolved knots embedded in a region of more diffuse emission. In this section we show that these regions can be interpreted as being (super) starburst regions (SSBs). An understanding of the physical nature of these regions is crucial for interpreting the UV emission from LBGs.

\subsection{Identification and Photometry}

In Figure 12 we indicate all the starburst regions identifiable by eye from the $U_{330}$ band images (small circles). Although our identification by eye is somewhat subjective, most regions are bright and isolated and thus easily recognized. We identify a total of 41 regions in our sample of eight galaxies. Some galaxies contain only one knot, while others contain as many as 10 . Next, we measured the fluxes and colors of each region using circular aperture photometry in matched $U_{330}$ and $z_{850}$ images (resolution of $\sim 0.12^{\prime \prime}$, FWHM). We were able to isolate all of the starburst regions identified in Figure 12 using circular apertures of $0.3^{\prime \prime}$ in diameter (corresponding to physical radii of $\sim 240-480 \mathrm{pc}$ ). The encircled energy (EE) measurements of Sirianni et al. (2005) show that circular apertures of this diameter enclose $\sim 80 \%$ of the total light of a point source observed with ACS. We did not apply the EE correction. Magnitudes were corrected for Galactic extinction, and the magnitude distribution of the 41 starburst regions is shown in Figure 13. Absolute magnitudes were calculated from $M=m-5 \log _{10}\left[D_{L}(\mathrm{pc})\right]+5+2.5 \log _{10}(1+z)$. We did not apply a $K$-correction as the central rest-frame wavelengths at which the absolute magnitudes are determined differ only by a small amount $(\lesssim 300 \AA)$. Figure 13 shows that our "by eye" selection is relatively complete at $m_{330} \lesssim 23 \mathrm{mag}\left(M_{330 /(1+z)} \lesssim-16.5\right)$. We also determined the flux and color of the large, diffuse region that 

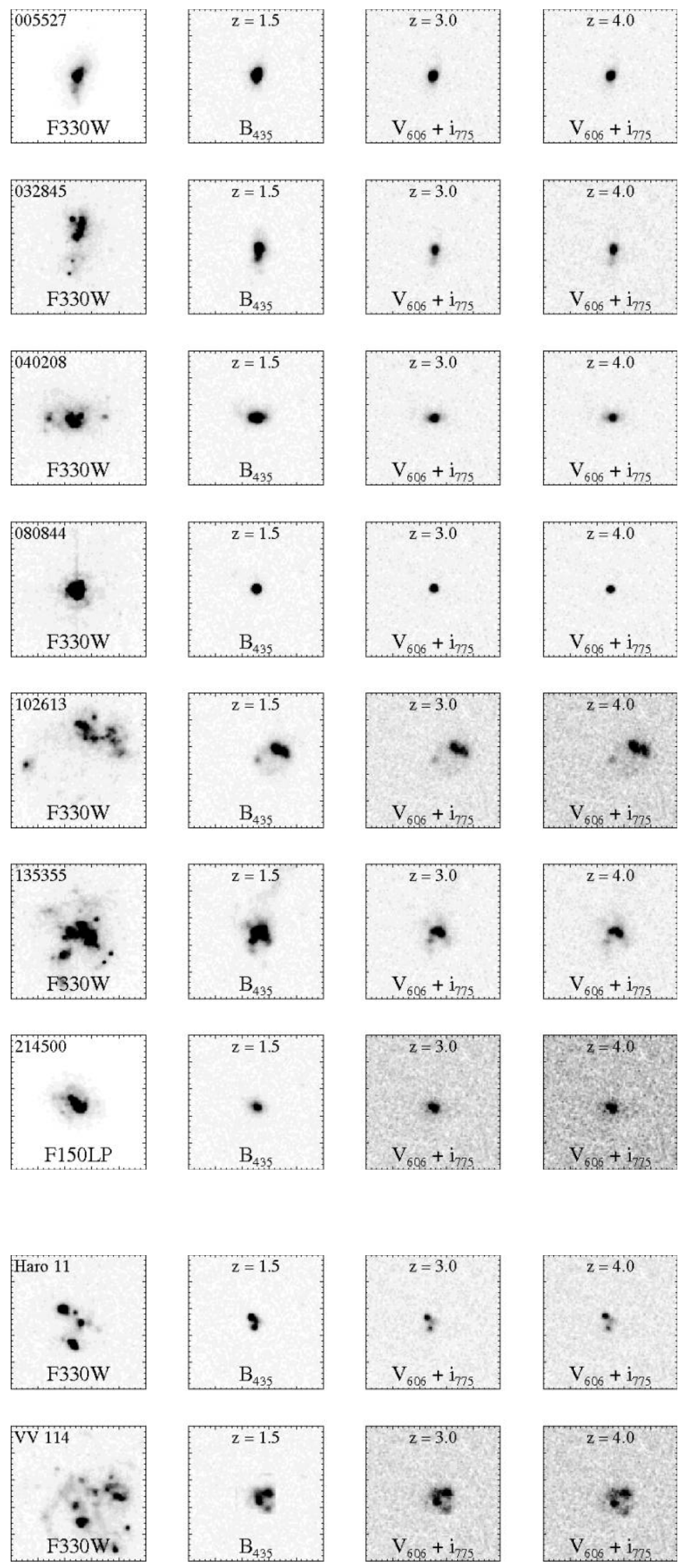

FIg. 7.- -Redshift simulations of the UVLG sample at the depth of GOODS ( $\$ 3.6 .2)$. Panels $\left(3^{\prime \prime} \times 3^{\prime \prime}\right)$ show the original, unredshifted HRC/F330W or SBC/F150LP images (left) and GOODS $B_{435}$ or $V_{606}+i_{775}$ simulations at $z=1.5$, 3.0, and 4.0. The last two rows show the redshift simulations of Haro 11 and VV 114 (§ 3.6.2) for comparison. 

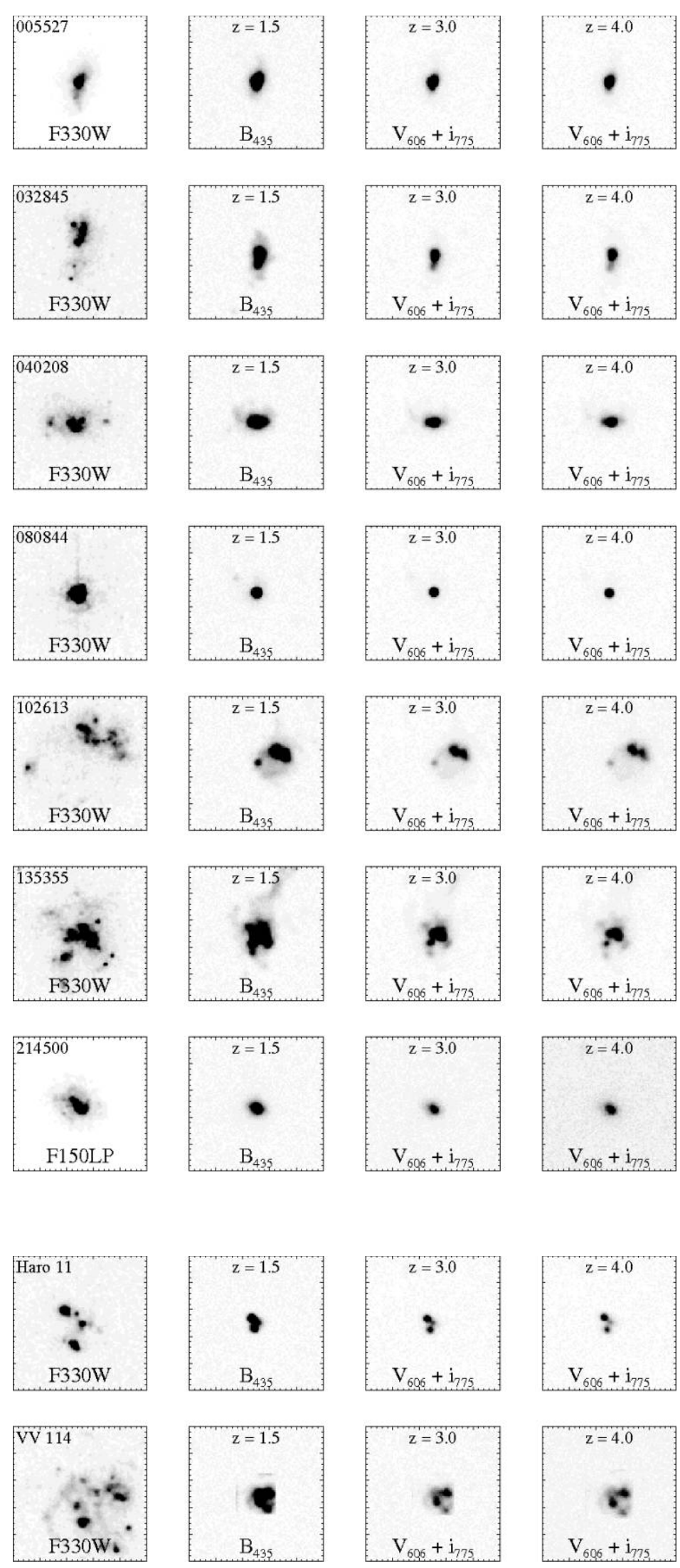

FIG. 8.- Redshift simulations at the depth of the UDF. See $\S 3.6 .2$ and the caption of Fig. 7 for details. 

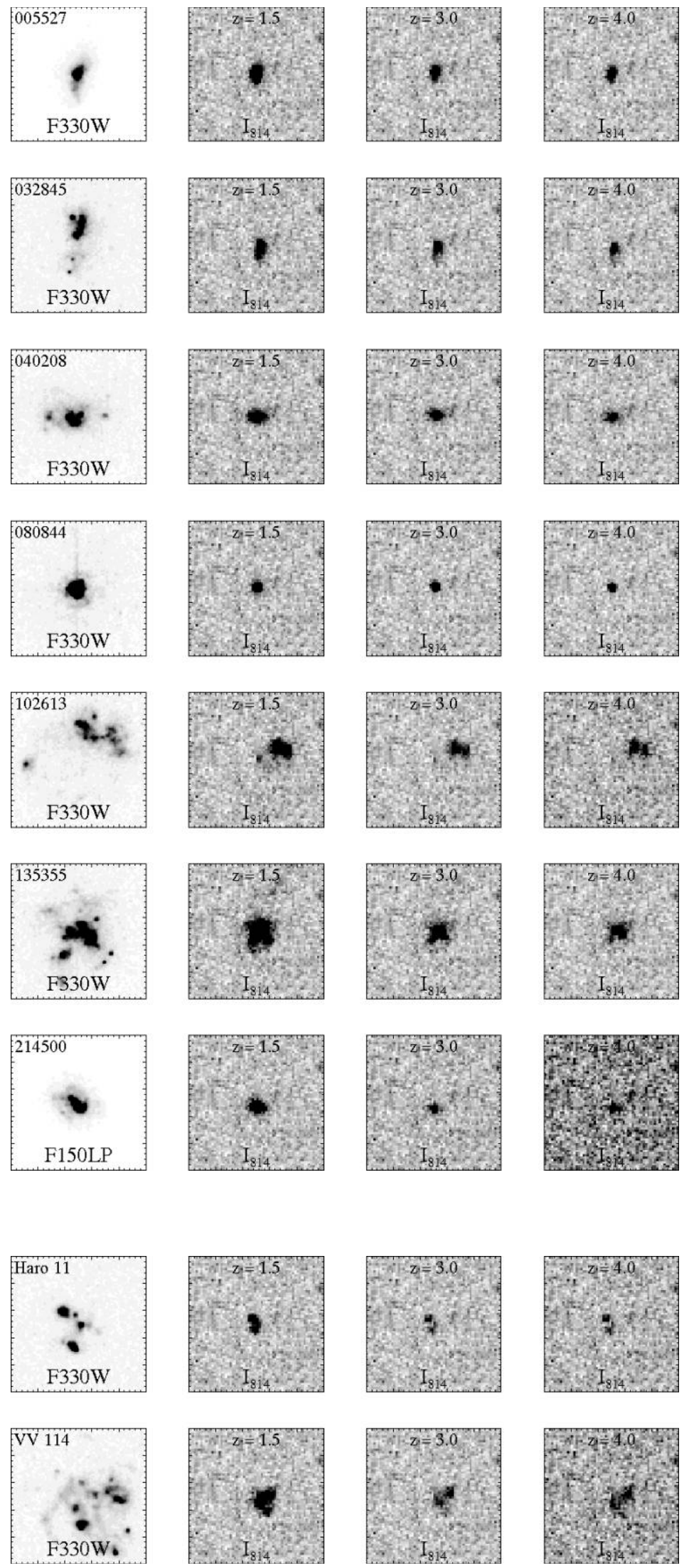

FIG. 9.- Redshift simulations at the depth of COSMOS. See $\S 3.6 .2$ and the caption of Fig. 7 for details. 

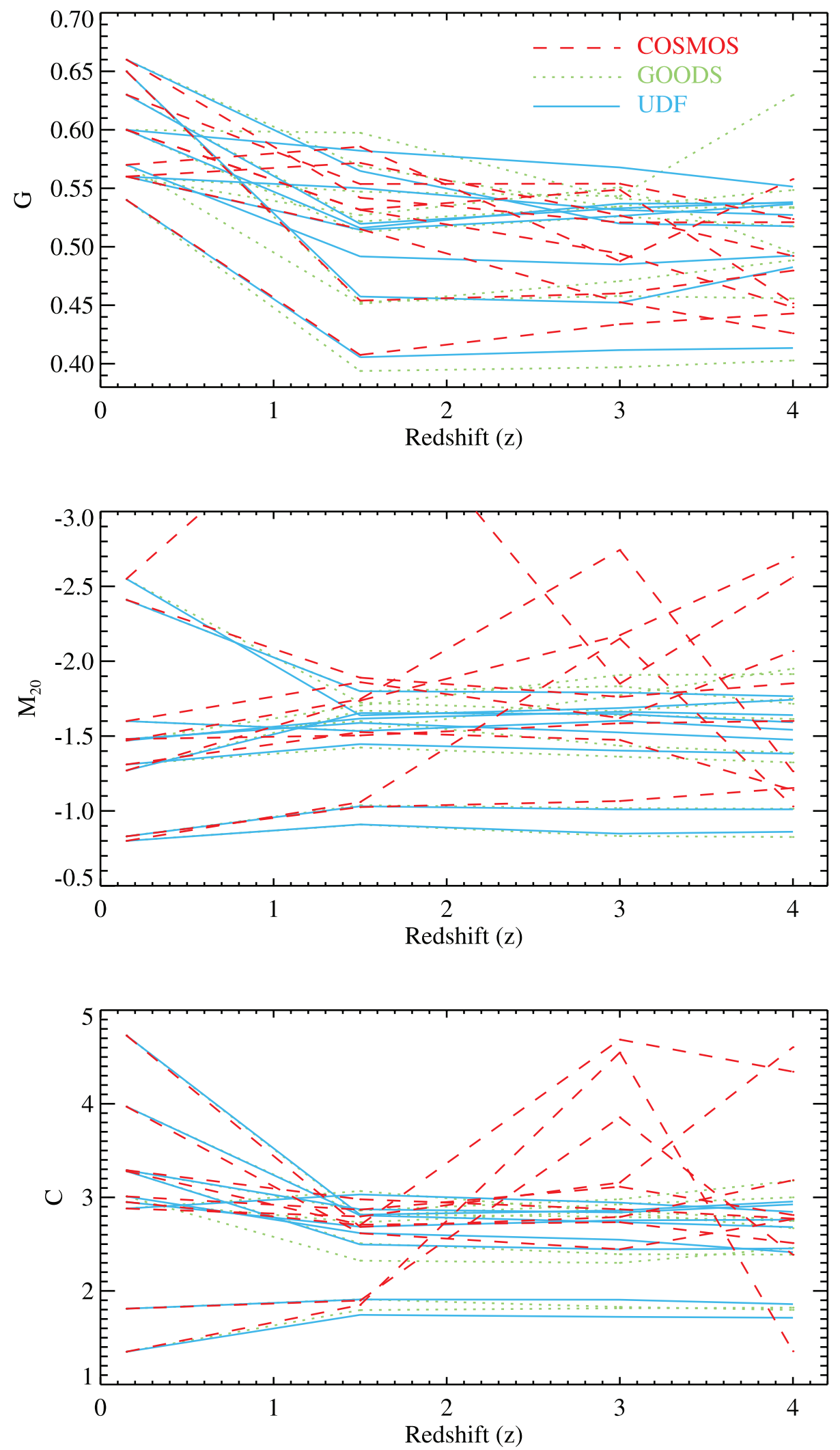

Fig. 10.- Morphological parameters of the UVLGs measured from the simulated images at different redshift. Objects were redshifted to $z=1.5,3$, and 4 and simulated at the depths of COSMOS (red dashed lines), GOODS (green dotted lines), and the UDF (blue solid lines). 

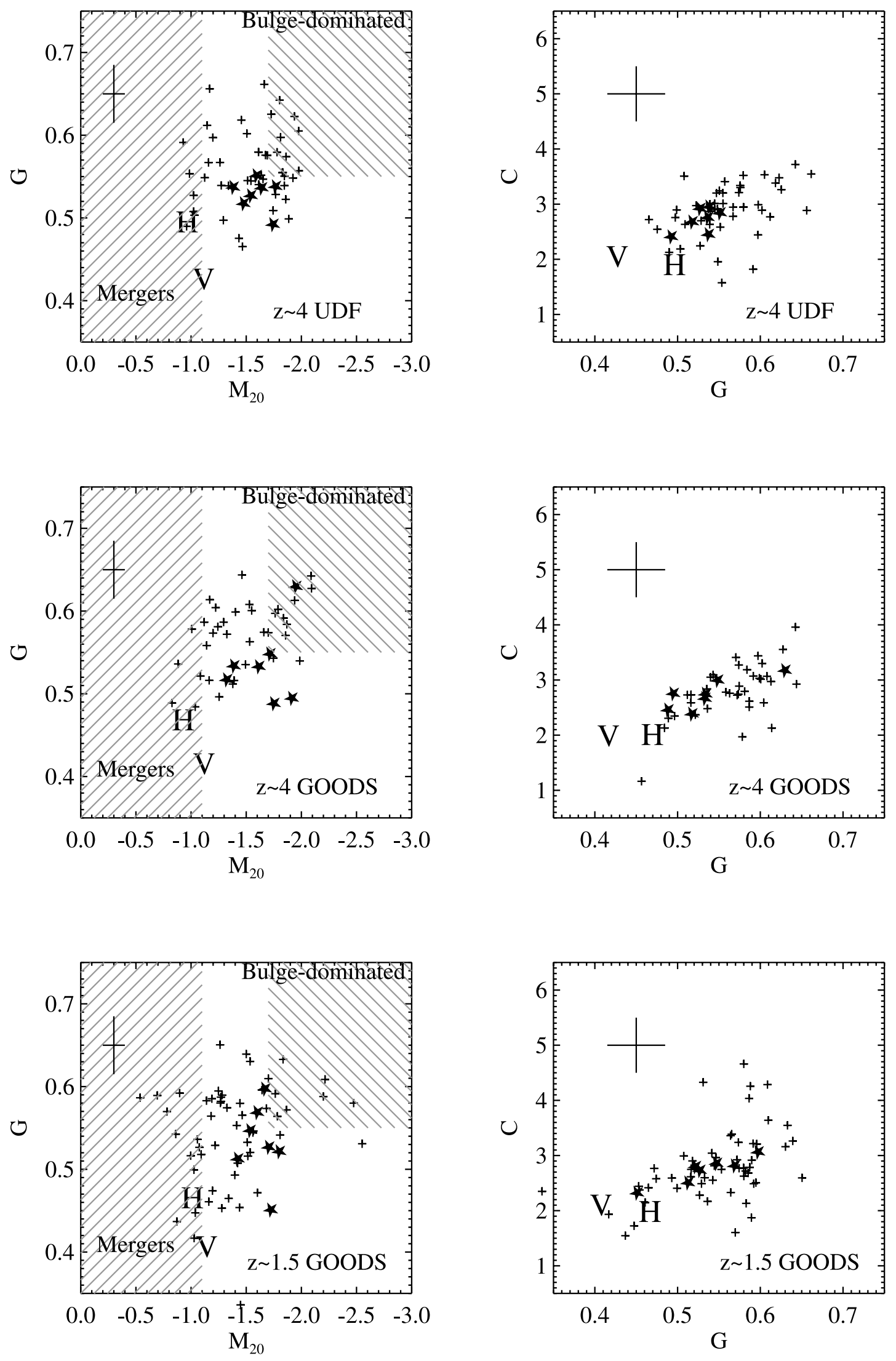

FIG. 11.-Comparison of the morphologies of LBGs at $z \sim 4$ and starburst galaxies at $z \sim 1.5$ from the samples of Lotz et al. (2006) ( plus signs) with the morphologies of the UVLGs (stars). The UVLGs were artificially redshifted to the mean redshift of the comparison samples and simulated at a similar depth. Top: Results for LBGs and UVLGs at the UDF depth at $z \sim 4$; middle: LBGs and UVLGs at the GOODS depth at $z \sim 4$; bottom: star-forming galaxies and UVLGs at the GOODS depth at $z \sim 1.5$. Haro 11 ("H") and VV 114 ("V") were simulated at the same redshift and depth as the UVLGs. For comparison, we have indicated the following (all adapted from Lotz et al. 2006): regions populated by likely mergers having multiple nuclei (left hatched area) and by bulge-dominated objects (right hatched area), and the typical errors for the high-redshift samples (large plus signs). In all panels, the UVLGs span a very similar range in parameter space compared to the comparison samples, indicating that UVLGs and LBGs have very similar quantitative morphologies. 

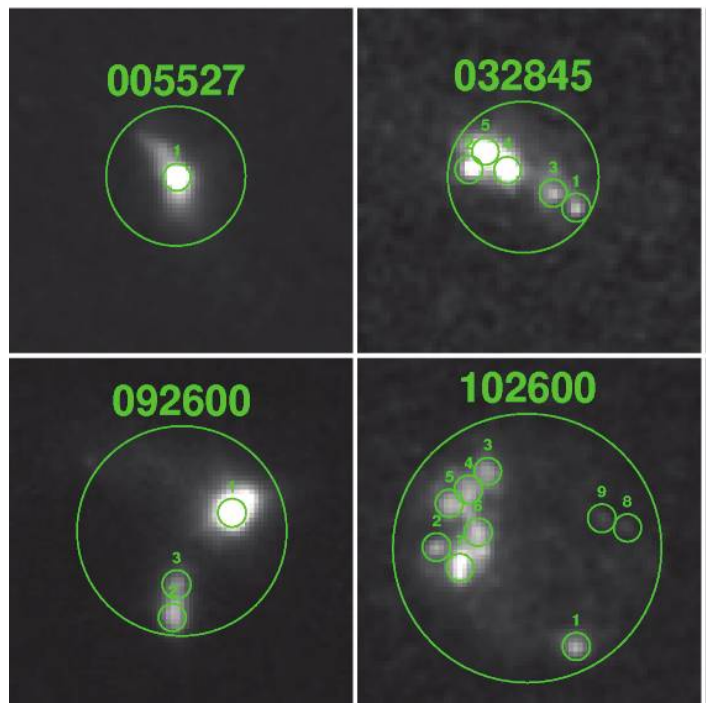

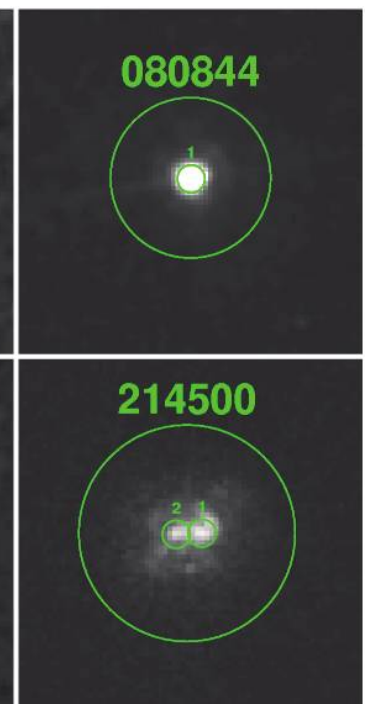

FIG. 12.- $U_{330}$ images indicating the apertures placed around the unresolved starburst regions identified by eye in each of the sources (small circles). Apertures measure $0.3^{\prime \prime}$ in diameter and are numbered for identification of each region in Fig. 14. Larger apertures were chosen to measure the average color of the regions in which the unresolved starbursts are situated (large circles). For object J214500 the image shown is $\mathrm{FUV}_{150}$. For completeness, we show the $z_{850}$ image for J092600 as no $U_{330}$ image is available.

generally surrounds the compact starburst knots (Fig. 12, large circles). These regions measure $\sim 2-5 \mathrm{kpc}$ in radius. The fluxes measured in the small apertures were subtracted from the larger aperture.

We find that the UV light is typically dominated by emission from the compact, luminous regions: the combined flux of the unresolved regions contributes an average of $\sim 50 \%$ to the total flux in $U_{330}$. Object $\mathrm{J} 102613$ has the lowest contribution from SSBs ( $\sim 34 \%)$ and object J080844 the highest ( 80\%). Although we do not have a $U_{330}$ image for J092600, we can infer that its structure is very similar to the other objects given that the unresolved knots contribute $\sim 42 \%$ to the total flux in $z_{850}$, and its morphology in $\mathrm{H} \alpha$ (assumed to be a good proxy for its $U_{330}$ morphology; see Fig. 2) shows a similar knotty structure.

\subsection{Color-Magnitude Diagram}

In Figure 14 we show the color-magnitude diagram of all regions identified in Figure 12. Objects J092600 and J214500 were omitted. Starburst regions from each galaxy are plotted using a different filled symbol, with their corresponding ID number from Figure 12 indicated for reference. Open symbols of corresponding shape indicate the diffuse regions that surround the compact regions. In order to compare the measured colors and magnitudes to model starburst tracks, we "dereddened" the measurements using the global reddening value for the stellar continuum that was derived from the bolometric dust-to-FUV luminosity ratio (see $\S 3.5$ ). We note that this correction is in general quite small. The compact regions span quite a large range both in color and in absolute magnitude (Fig. 14, filled symbols). The diffuse regions, defined by the larger areas in Figure 12 but with the circular regions around all the identified point sources removed, lie at $M_{U} \sim$ -20 and $\left(U_{330}-z_{850}\right) \sim 0.5$ (Fig. 14, open symbols).

To interpret these colors, we have used Starburst99 (Leitherer \& Heckman 1995; Leitherer et al. 1999) to predict the color and magnitude evolution of a starburst as observed through our filters. The tracks in the left panel of Figure 14 represent an instantaneous burst model with burst masses of $M=10^{7}$ and $10^{8} M_{\odot}$, while tracks in the right panel indicate a continuous star formation model having SFRs of $0.1,1$, and $5 M_{\odot} \mathrm{yr}^{-1}$. We use a Kroupa initial mass function (IMF) with a slope of $\alpha=1.3$ between 0.1 and $0.5 M_{\odot}$ and $\alpha=2.3$ between 0.5 and $100 M_{\odot}$. We also use the 1994 Padova models with thermally pulsating asymptotic giant branch stars added (Vázquez \& Leitherer 2005). The tracks shown were obtained after redshifting the rest-frame spectra to $z=0.15$, the average redshift of our sample, and measuring the magnitudes in $U_{330}$ and $z_{850}$. The blue lines correspond to a metallicity equal to
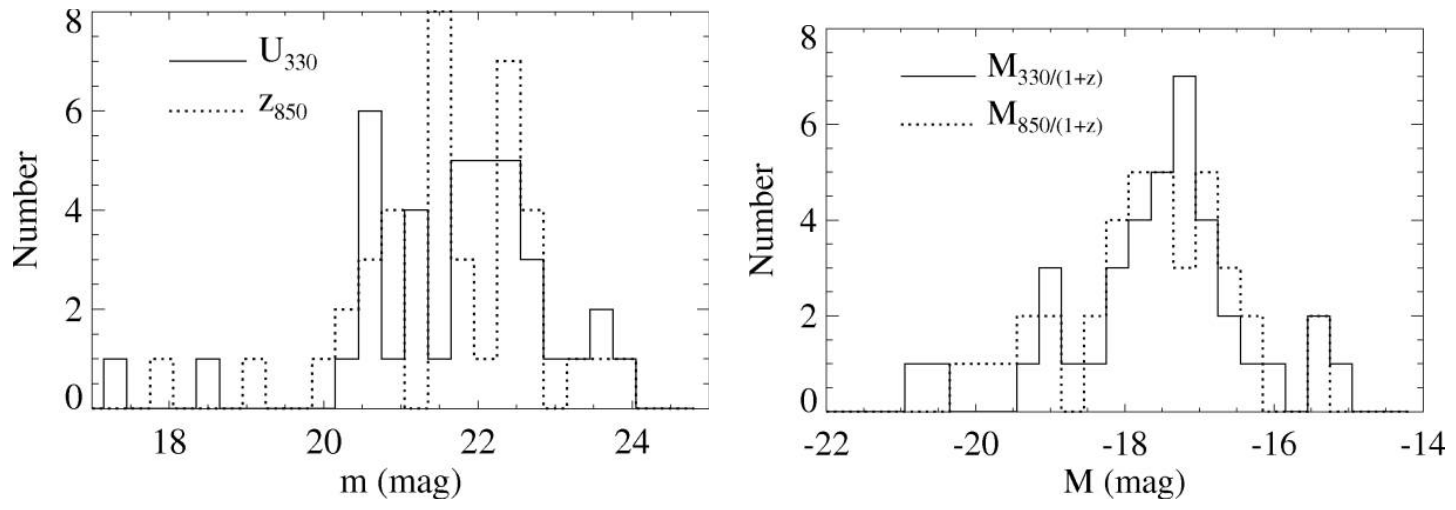

FIG. 13.-Apparent (left) and absolute (right) magnitude distributions in $U_{330}$ (solid lines) and $z_{850}$ (dotted lines) of the unresolved starburst regions identified in Fig. 12. The magnitudes were extinction corrected using a global reddening value derived from the bolometric dust-to-FUV luminosity ratio (see Table 3 ). 

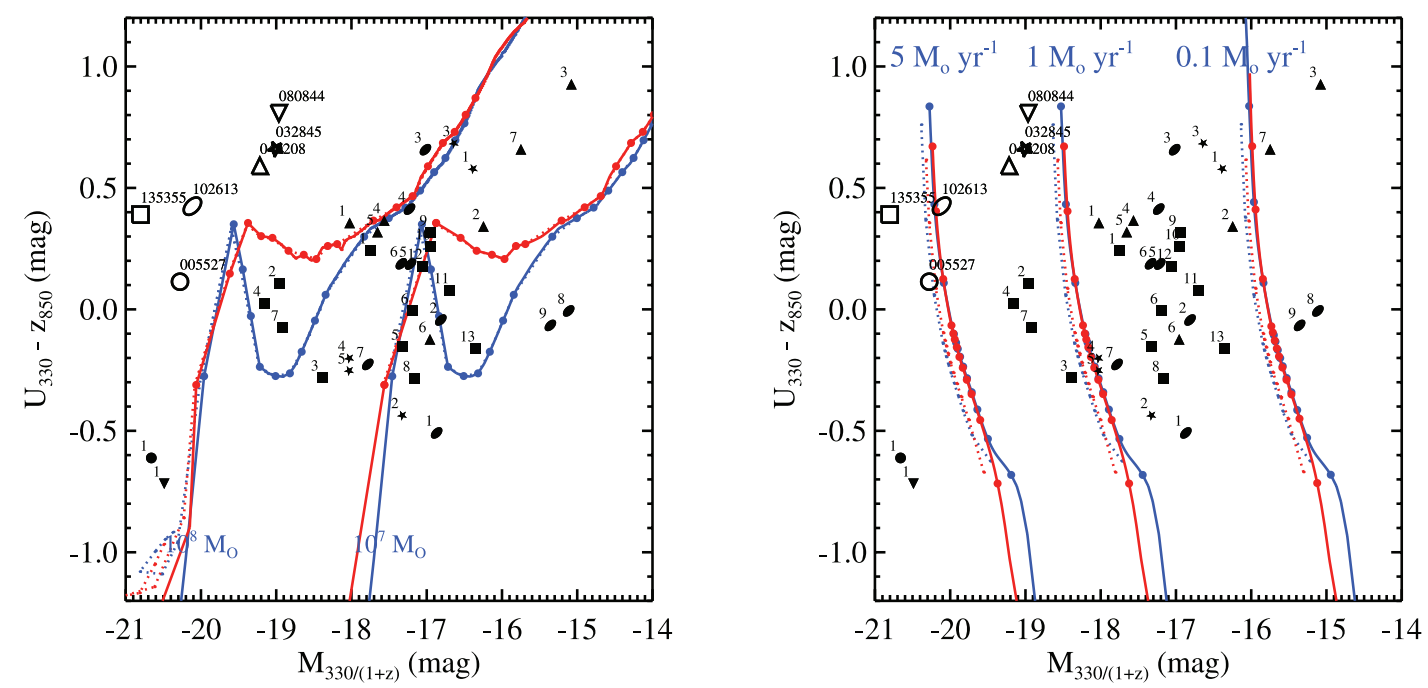

FIG. 14. - Color-magnitude diagram of the individual starburst regions identified in Fig. 12. Filled, numbered symbols correspond to the unresolved regions identified in J005527 (circle), J032845 (star), J040208 (upward-pointing triangle), J080844 (downward-pointing triangle), J102613 (oval), and J135355 (square). The large, open symbols correspond to the large apertures in Fig. 12 with the point sources removed. Tracks indicate the color and magnitude evolution of an instantaneous burst model (left) and a continuous star formation model (right) simulated using Starburst99. Blue tracks are for LMC metallicity $(Z=0.008)$, red tracks for solar metallicity $(Z=0.02)$. For instantaneous bursts we plot tracks having a burst mass of $10^{8}$ and $10^{7} M_{\odot}$. For continuous models we plot tracks having SFRs of 5, 1 , and $0.1 M_{\odot} \mathrm{yr}^{-1}$. Ages in Myr have been indicated along the tracks (marked at 6, 7, 8, 9, 10,12,14,16, 18, 20,30, 40, 50, 60, 70, 80, 90, and 100 Myr for the burst models and at 10, 20, 30, 40, 50, 60, 70, 80, 90, 100, 200, 500, and $1000 \mathrm{Myr}$ for the continuous models). Dotted lines indicate the colors if we include the contribution from the nebular continuum. The model tracks are reddening-free, but the observed colors and magnitudes were dereddened using a global reddening value derived from the bolometric dust-to-FUV luminosity ratio (see Table 3). The starburst regions of J214500 were omitted because of the different filter set used during those observations. Object J092600 was omitted because no $U_{330}$ is available.

that of the Large Magellanic Cloud $(Z=0.008)$, while the red lines are for solar metallicity. Ages (in Myr) have been indicated along the tracks. Dashed lines indicate the contribution from the nebular continuum (assuming no reddening). The general behavior of all tracks is to become redder with age. After about $10 \mathrm{Myr}$, the colors become steadily redder because the most massive O stars have become supernovae and lower mass $\mathrm{O}$ stars have become red supergiants. The red supergiant-dominated phase at $10-20 \mathrm{Myr}$ is highly dependent on metallicity, as their number is lower and their temperature is warmer in lower metallicity starbursts (Vázquez \& Leitherer 2005). After $20 \mathrm{Myr}$, O stars have disappeared, leading to a gradual reddening with age. Continuous models become brighter, while instantaneous models fade with time.

\subsection{Results}

The instantaneous burst and continuous star formation models have been chosen to bracket the range of possible star formation histories of our compact starburst regions. Most of the regions are clearly detected in $\mathrm{H} \alpha$, indicating that they must at least have had very recent star formation. In Figure 15 we plot the SSBs in the plane of color versus the rest-frame equivalent width (EW) of $\mathrm{H} \alpha$ measured for each SSB. The measured EWs are 30-500 $\AA$ and are seen to correlate with the $U_{330}-z_{850}$ color, suggesting that the $\mathrm{H} \alpha \mathrm{EW}$ is a reasonably good age indicator. We have overplotted the predicted EW of $\mathrm{H} \alpha$ based on the Starburst99 models shown in Figure 14. For instantaneous models, the $\mathrm{H} \alpha \mathrm{EW}$ is less than $\sim 100 \AA \AA$ after $6 \mathrm{Myr}$ and less than $10 \AA$ after only $10 \mathrm{Myr}$ (dashed lines). For continuous star-forming models, a high $\mathrm{H} \alpha$ $\mathrm{EW}$ is maintained over a much longer period of time, with $\mathrm{EW}_{\mathrm{H} \alpha} \gtrsim$ $100 \AA ̊$ for ages $\lesssim 1$ Gyr.

Comparison of the instantaneous burst and continuous star formation tracks with the observed compact and diffuse regions led us to the following conclusions:

1. The $M \simeq 10^{7}-10^{8} M_{\odot}$ instantaneous starburst tracks shown in the left panel of Figure 14 provide a generally good match to the majority of the compact regions, but the ages of $\sim 10-100 \mathrm{Myr}$ inferred are inconsistent with their large $\mathrm{H} \alpha \mathrm{EWs}$, as shown in Figure 15. A better match to both the color-magnitude diagram and the $\mathrm{H} \alpha$ EWs is provided by the continuous star formation models shown in the right panel of Figure 14. The exact ages are uncertain because of small-scale differences between the $U_{330}$ and $\mathrm{H} \alpha$ morphologies (note that the apertures were defined on the $U_{330}$ image), uncertainties in the small-scale dust distribution

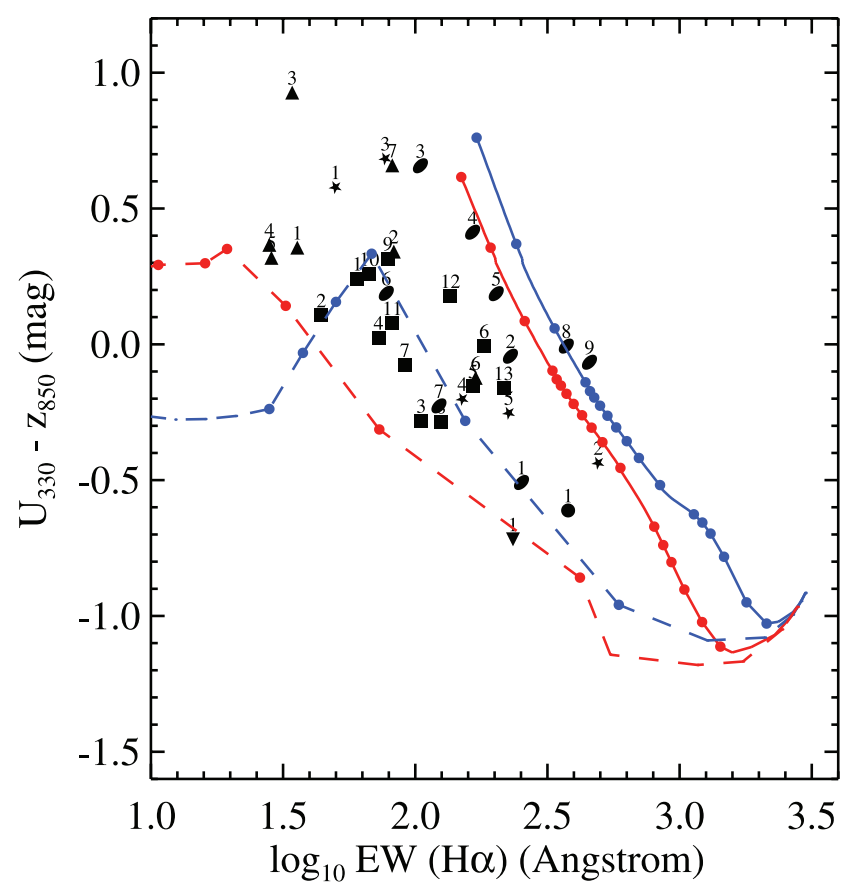

FIG. 15.- Rest-frame equivalent width $\mathrm{H} \alpha$ vs. $U_{330}-z_{850}$ color for the SSBs. Tracks indicate the age evolution of the $\mathrm{H} \alpha \mathrm{EW}$ for instantaneous burst (dashed lines; with ages marked at 5, 6, 7, 8, 9, and $10 \mathrm{Myr}$ ) and continuous star formation (solid lines; with ages marked at 5, 6, 7, 8, 9, 10, 20, 30, 40, 50, 60, 70, 80, 90, $100,200,500$, and $1000 \mathrm{Myr}$ ) models from Starburst99. See text and the caption of Fig. 14 for further details. 
(now assumed to be a single foreground screen), and because of the unknown fraction of the light that is due to the background population inside each of the small apertures (not subtracted). Our best estimates for the ages of the starburst regions range from a few tens to a few hundreds of Myr based on a comparison with the tracks in Figure 14. These ages should be regarded as upper limits because we may have underestimated the contribution from dust and older stars. The scatter in $M_{U}$ magnitude can be interpreted as a sequence in SFR ranging from $\sim 0.1$ to $\sim 5 M_{\odot} \mathrm{yr}^{-1}$ (or total burst mass ranging from $\sim 10^{7}$ to several times $\left.10^{8} M_{\odot}\right)$. The scatter in $\left(U_{330}-z_{850}\right)$ color can be interpreted as a sequence in age (or burst age in the case of instantaneous bursts). Note that our results are almost independent of metallicity.

2. For the brightest starburst regions, there is a strong tendency for neighboring regions to have a very similar color and magnitude, e.g., regions $(2,4,7)$ in object J135355, regions $(1,4,5)$ in object J040208, and regions $(4,5)$ in object J032845. This suggests that the starburst in these objects tends to occur in the form of several coeval and equally massive bursts.

3. The two compact regions that are outliers (in the sense that they appear to be both extremely young, $\sim 6 \mathrm{Myr}$, and very massive, $M_{*} \sim 10^{8} M_{\odot}$ ) each correspond to objects where the UV emission is dominated by a single, luminous knot (objects J005527 and J080844). Both objects have a very large $\mathrm{H} \alpha \mathrm{EW}$, indicating that they are massive, young starbursts. The SDSS spectrum ${ }^{12}$ of J005527 shows a broad feature around a bright and narrow He II $\lambda 4686$ line that is characteristic of Wolf-Rayet (WR) galaxies (e.g., Conti 1991; Sargent \& Filippenko 1991; Izotov et al. 1997). The WR phase is a relatively short phase in the life of the most massive stars, during which they experience a large mass loss. The presence of WR stars is a unique indicator of young starbursts, as WR stars disappear after $\sim 6-10 \mathrm{Myr}$ depending on the metallicity of the burst, with less mass loss occurring at lower metallicity (Leitherer \& Heckman 1995). Further confirmation of this young age comes from the large equivalent width of $\mathrm{H} \beta$ $(\sim 60 \AA)$, consistent with an age of $\sim 6 \mathrm{Myr}$ (Leitherer \& Heckman $1995)$.

4. Some other regions with very blue colors and high EW in $\mathrm{H} \alpha$ (e.g., region 2 in J032845 and region 1 in J102613) appear to be as young as the previous two but are $\sim 2-3$ mag fainter.

5. The more diffuse, inner regions in which the SSBs are embedded (Fig. 14, open symbols) are typically a few tenths of a magnitude redder than the average color of the compact SSBs they surround, and their total magnitudes are very similar to the combined flux of their SSBs. These regions contain an older stellar population. Given their large physical extent, the instantaneous burst model is unlikely to be appropriate. We find that the colors and luminosities of these regions are in good agreement with continuous star formation models with SFRs of $\simeq 1-5 M_{\odot} \mathrm{yr}^{-1}$ and ages of $\simeq 0.2-1.0 \mathrm{Gyr}$.

6 . The outer annuli (defined as the region between $r_{50,850}$ and $r_{90,850}$; see Table 4 ) are consistent with older populations having ages between a few Gyr and a Hubble time, depending on the star formation history.

\subsection{Summary}

The starburst regions observed in the local LBG analogs are highly compact (radii of 100-300 pc) and characterized by bright, unresolved knots of emission within a larger region of diffuse star formation that extends out to a radius of a few kiloparsecs. The total UV emission from these compact starburst regions is sub-

12 See http://cas.sdss.org/dr5/en/tools/explore/obj.asp?id=588015508738539596. stantial, indicating that $30 \%-80 \%$ of the total SFR is generated in these knots. Comparison with Starburst99 evolutionary tracks indicates that some of the regions are due to very young ( $\sim 6 \mathrm{Myr}$ ) bursts, while other regions may have been forming stars in a more continuous manner for several tens to several hundreds of Myr as indicated by their relatively red colors and large $\mathrm{H} \alpha$ equivalent widths. There is a tendency for neighboring knots to have similar colors and luminosities. This implies that they may be coeval and of similar mass. Most of the SSBs are still unresolved in the unbinned $U_{330}$ images $\left(\approx 0.075^{\prime \prime}\right.$, FWHM). The stellar mass densities implied are $\sim 10^{2}-10^{3} M_{\odot} \mathrm{pc}^{-2}$ estimated from their masses of $\sim 10^{7}-10^{8} M_{\odot}$ and assuming an effective radius of $\sim 100 \mathrm{pc}$.

The masses of the SSBs are 1-2 orders of magnitude larger than the masses of the most massive clusters found in the local universe. We may therefore conclude that the (unresolved) SSBs seen in Figure 12 are likely composed of smaller units. Meurer et al. (1995) found that typical starburst regions in local starburst galaxies consist of diffuse stars and clusters in the ratio of, respectively, $80 \%$ to $20 \%$. The most prominent of the clusters, the socalled super star clusters (SSCs), have masses of $\approx 10^{5}-10^{6} M_{\odot}$ and sizes of a few to $\sim 10 \mathrm{pc}$ and likely correspond to globular clusters in the process of formation (provided that the stellar IMF extends down to $0.1 M_{\odot}$ ).

We can get an even better idea of the subresolution structure of the SSBs by comparing the starburst regions in Haro 11 and VV 114 shown in the top panels of Figure 6 with the same regions simulated at $z=0.15$ shown in the bottom panels of the same figure. The majority of the bright nuclei seen in the images simulated at $z=0.15$ are a blend of numerous smaller SSCs that can clearly be seen in their unredshifted images. However, the most northern and brightest knot in the redshifted image of Haro 11 is still largely unresolved in its unredshifted image. This indicates that single, highly massive star clusters are capable of dominating the rest-frame UV/optical morphology of LBG-like galaxies at virtually any redshift. A detailed determination of the sizes, masses, and ages of the star clusters in Haro 11 and VV 114 is beyond the scope of this paper and can be found elsewhere (Scoville et al. 2000; Hayes et al. 2007; A. Adamo et al. 2008, in preparation).

\section{DISCUSSION}

\subsection{Triggering Mechanism}

Although the number of objects in our HST sample is currently quite small, the results presented in this paper can nevertheless guide us in the interpretation of the morphologies of these local starburst galaxies and, by extension, those of LBGs at higher redshifts.

Are the morphologies evidence for merging? - Although some of the SDSS images showed evidence for close companions or faint extended emission, the galaxies were extremely compact and it was not at all clear from the SDSS images that they were highly disturbed. The biggest surprise from the $H S T$ images was that most of the UVLGs show disturbed morphologies at scales well below the SDSS seeing or sensitivity. Each UVLG shows at least one of the typical signs of merging or interaction, such as multiplicity or twists in the faint, outer isophotes (e.g., J005527, J032845, J040208, J092600), tidal debris or tails (e.g., J032845, J102613, J214500), and close companions (e.g., J080844, J102613, J135355, J214500).

While none of the UVLGs appear as fully formed Hubble sequence galaxies, in one case, J214500, a small spiral structure seems to be present both in the FUV and in $V_{606}$. Nonetheless, even in this object there is circumstantial evidence that the starburst was triggered by a merging event, as evidenced by its companion 
that appears to have gone straight through its nucleus, leaving behind a trail of tidal debris. The projected distance between the centroid of the starburst region and the companion is about $5 \mathrm{kpc}$. The companion galaxy would traverse this distance in $\sim 10-$ $100 \mathrm{Myr}$ assuming a velocity of a few hundred kilometers per second and a straight trajectory in the plane of the sky. If we assume that the starburst is triggered by the interaction (as suggested by the $V_{606}$ morphology), this timescale constrains the maximum possible age of the burst. The results are consistent with 30-100 Myr ages derived from the colors and magnitudes of these systems. In many of the other cases, the region that dominates the UV morphology often lies at the interface of two diffuse merging structures, as identified by the contours of their outer isophotes in $z_{850}$. In the remaining cases, a companion is always seen at a distance of less than $\lesssim 5 \mathrm{kpc}$ from the UVLG. It is well known that close neighbors can affect the SFR in galaxies, with close pairs having separations of $\$ 50 \mathrm{kpc}$ showing enhanced star formation (e.g., Larson \& Tinsley 1978; Lambas et al. 2003 ; Nikolic et al. 2004; Woods et al. 2006; Owers et al. 2007; Li et al. 2008). This supports the idea that the starbursts are linked to mergers. The merging galaxies must be very gas-rich in order to trigger the very high level of enhanced star formation and the luminous SSBs that we observe.

It has been proposed that starburst galaxies at high redshift, such as LBGs, are triggered by mergers of relatively gas-rich disk galaxies (Somerville et al. 2001). Simulations indicate that mergers of gas-rich galaxies can trigger massive starbursts. The conditions of the starburst depend on a number of key parameters of the merging system (see, e.g., Mihos \& Hernquist 1994a, 1994b; Springel \& Hernquist 2005; di Matteo et al. 2007 and references therein). During a merger of disk galaxies, barlike patterns develop that efficiently funnel gas into the central region. The gas flow to the center is generally not disrupted or depleted due to star formation during the early stages of the merger, although the inflow of gas can be limited due to resonances if a bulge component is present, reducing the central gas densities and overall SFR and duration of the central starburst. The presence of gas is not always a sufficient condition for generating a starburst. The star formation efficiency of a merger depends largely on the galaxy spin direction (retrograde encounters have higher efficiency) and on the amount of gas that is being expelled due to the tidal interaction at first passage (Springel \& Hernquist 2005; di Matteo et al. 2007).

We remind the reader that typical $\left(L \sim L_{z=3}^{*}\right)$ LBGs are not dwarf galaxies, but have masses of $\sim 10^{10} M_{\odot}$ and SFRs that are much higher than those of typical (unobscured) local starbursts (Papovich et al. 2001; Shapley et al. 2001). They do not show evidence for spiral structure (Papovich et al. 2005). As such, they are very similar to our local LBG analogs. We conclude that the main mechanism responsible for triggering star formation in both high-redshift LBGs and their local analogs is likely to be mergers.

Are the compact SSBs triggered by the mergers? - Luminous star clusters (e.g., SSCs) occur in many irregular and dwarf galaxies (e.g., 30 Dor in the LMC). The triggering of compact SSBs as massive as the ones in our sample of UVLGs probably requires a highly efficient inflow and compression of the gas that is typically only seen in galaxy mergers or interactions. The inflow ensures that the pressure in the interstellar medium becomes larger than the internal pressure of giant molecular clouds and that the clouds can collapse and form SSCs before they are disrupted by supernova explosions (Bekki 2004). Smoothed particle hydrodynamics simulations of galaxy mergers suggest that the properties of the starburst are determined by the magnitude, timescale, and geometry of this inflow (see Mihos \& Hernquist 1994a; Bekki
\& Couch 2001; Bekki 2004; di Matteo et al. 2007). Mihos \& Hernquist (1994a) found that the central starbursts resulting from gas-rich mergers can have total sizes of $\sim 350 \mathrm{pc}$ and contain most of the total mass in new stars. Bekki \& Couch (2001) predict that (1) SSCs have a narrow age distribution ( $200 \mathrm{Myr})$ because they form most efficiently during the stage when the gas density is the highest, (2) the total number and intrinsic masses of SSCs are larger in major mergers due to larger tidal effects and higher peak SFRs, and (3) SSC production is more efficient in merging galaxies (e.g., ULIRGs; Sanders \& Mirabel 1996) than in tidally interacting galaxies (e.g., M82; Kennicutt 1998).

Observations show that starbursts indeed occur in regions where the gas densities are the highest, typically in the cores (e.g., Arp 220; Shaya et al. 1994) and in circumnuclear starburst regions (e.g., VV 114; Scoville et al. 2000). Stars may form in large complexes having total stellar masses of $\sim 10^{7}-10^{9} M_{\odot}$ and containing several tens to hundreds of SSCs, as well as smaller star clusters. This is true both in ULIRGs and in compact blue galaxies (e.g., Meurer et al. 1995; Östlin et al. 1998; Whitmore et al. 1999; Zepf et al. 1999; Goldader et al. 2002 and references therein).

We conclude that the characteristic morphologies, ages, sizes, and masses of the starburst regions we have observed are consistent with those predicted by simulations of gas-rich mergers. They are also analogous to the large star-forming complexes observed in very nearby merging galaxies.

\subsection{Low-Redshift Lessons for High-Redshift LBGs}

Because of their relative proximity, studies of local star-forming galaxies can help us understand the nature of starburst galaxies at high redshifts. As explained in $\S 1$, up to now these comparisons have been of limited usefulness because typical local starburst galaxies are systematically different from LBGs at high redshift. The sample of local LBG analogs first studied by H05 was specifically designed to bridge this gap. In this paper we have studied the detailed morphologies of these systems, and we now discuss how our results impact the study of star-forming galaxies at high redshifts.

\subsubsection{Morphologies and Sizes}

Our analysis of the HST data has established that UVLGs have a light distribution dominated by very compact starbursting regions (ranging in number from 1 to more than 10 per galaxy) that are triggered by mergers or interactions. In most cases, an older, diffuse, stellar component is also present. Our redshift simulations showed that the sizes and morphologies at $z=1.5-4$ are similar to those of LBGs (e.g., Giavalisco et al. 1996b; Conselice 2006; Lotz et al. 2006; Overzier et al. 2008; Younger et al. 2007). The morphological parameters measured at rest-frame UV and optical wavelengths are very similar. This is unlike typical local galaxies but very similar to LBGs as both are dominated by young stars (e.g., Papovich et al. 2005).

The redshift simulations presented in $\oint 3$ yield a number of important conclusions: (1) at high redshift, the compact starburst regions can blend, giving rise to a galaxy image that appears to have the one or two bright "knots" with a total half-light radius of $1-2 \mathrm{kpc} ;(2)$ the diffuse emission is partly lost in the background noise or may blend with the knots; and (3) the undegraded optical images were in most cases required to establish a definite connection between the (UV) starbursts and merging ( $§ 5.1$ ).

If knots are present in LBGs, they are typically not resolved in $H S T$ observations, ground-based spectroscopy with adaptive optics (resolution $\sim 0.1^{\prime \prime}$ or $\sim 1 \mathrm{kpc}$; Genzel et al. 2006; Law et al. $2007 \mathrm{a}$ ), or standard spectroscopy (resolution $\sim 0.5^{\prime \prime}$ or $\sim 4 \mathrm{kpc}$; 
Förster Schreiber et al. 2006; Erb et al. 2006b). Because imaging observations of high-redshift LBGs lack the sensitivity and resolution to see the (often subtle) features associated with merging, two- or three-dimensional spectroscopy is the most promising method of studying the connection between star formation and morphology in these objects. Small samples studied to date show complex kinematics and high nuclear gas fractions that are consistent with the merger hypothesis (Erb et al. 2003, 2006b; Förster Schreiber et al. 2006; Law et al. 2007a). However, some starforming objects at $z \sim 2$ may have more ordered underlying structures (Förster Schreiber et al. 2006; Genzel et al. 2006; Wright et al. 2007).

Coming back to the important, long-standing question of whether the irregular morphologies of LBGs are a sign of merging or of patchy star formation within a single (forming) disk (e.g., Law et al. 2007b), it is safe to say that in virtually every single case presented in this paper we do not see any direct evidence for merging based on the HST UV images alone. However, in virtually every single case evidence for merging is readily apparent from the rest-frame optical image, and we reiterate that most of the features suggestive of merging would be too faint or too small to be seen at $z \sim 2-4$. In some of the local LBG analogs the burst occurs predominantly in only one of the members of a merging or interacting pair. In others the merger seems to be in such an advanced state that one cannot speak about separate systems any more, making the distinction between "merging" and "patchy disk" essentially irrelevant. We conclude that the UV morphologies of LBGs are likely patchy as the result of merging.

Although both the UV and optical morphologies are dominated by compact starbursts, some of our objects show evidence for an older, extended component, which is generally not seen in highredshift LBGs (e.g., Papovich et al. 2005). In this respect, the UVLGs may be more evolved than LBGs at high redshift. However, it is important to remember that the age of the universe at $z \sim 3$ is only $\sim 2$ Gyr. The spectral energy distributions (SEDs) of LBGs are consistent with the presence of such a "maximally old" stellar component (Papovich et al. 2001). The limiting surface brightness of rest-frame optical images of LBGs in the NICMOS HDF-N indicates that these features are likely often too faint to be detected. Also, the rest-frame wavelength of the reddest NICMOS images of LBGs $\left(H_{160}\right.$ band) corresponds to only about half that of our $z$-band observations of the UVLGs and so is less sensitive to detection of an older population. Resolution effects could play a role as well. In Paper II we will carry out redshift simulations of a large set of rest-frame optical images of UVLGs to investigate these optical structures in detail.

Because high surface brightness regions in LBGs are very likely to be blends of SSBs, we stress that one must be cautious in interpreting size/morphology measurements in terms of disklike or bulgelike components. The half-light or effective radius may be more directly related to the typical radius over which the compact SSBs are distributed, rather than to the scale size of a (forming) bulge or disk (see also Noguchi 1999; Immeli et al. 2004; Law et al. 2007a), although the two are likely closely related if stars predominantly form inside these burst regions and relaxation or tidal disruption spreads them out over time. Although we do not rule out the possibility that some fraction of LBGs has bulges at redshifts as high as $z \sim 4$, the high physical resolution data presented here suggest that this is possibly far less common than currently believed based on morphological parameters (e.g., Lotz et al. [2006] estimate $~ 30 \%$ ).

Evidence that the small-scale structure of LBG knots is indeed of the nature advocated in this paper is found in a few rare cases where LBGs are observed at high magnification due to gravita- tional lensing. By studying a lensed galaxy at $z=4.92$, Franx et al. (1997) were the first to show that high surface brightness SSBs having sizes of a few hundred parsecs form an essential contribution $(\sim 75 \%)$ to the UV flux in LBGs. Similar features can be seen in other lensed systems that have been discovered at high redshift (Bunker et al. 2000; Ellis et al. 2001; Smail et al. 2007; Swinbank et al. 2007). We note that some fraction of LBGs with very knotty structures may be discarded in current large surveys because their light profiles are similar to those of stars. Our sample indicates that this number could be on the order of $13 \%-25 \%$ (one to two out of eight objects). If we compare the substructure of the SSBs in Haro 11 and VV 114 at $z=0.02$ with the same regions simulated at $z=0.15$ (Fig. 6), we see that the majority of the bright nuclei seen in the images simulated at $z=0.15$ are a blend of smaller SSCs identified in their unredshifted images. However, the most northern and brightest knot in the redshifted image of Haro 11 is still largely unresolved in its unredshifted image, indicating that single, luminous star clusters are capable of dominating the rest-frame UV/optical morphology of LBG-like galaxies at virtually any redshift.

As shown by Dahlen et al. (2007), the fraction of star-forming galaxies having a bulgelike morphology at rest frame $2800 \AA \mathrm{de}$ creases from $\sim 30 \%$ at $z \approx 2$ to $\sim 10 \%$ at $z \approx 0.5$, illustrating the relative importance of concentrated star formation at high redshift compared to low redshift. Zirm et al. (2007) have pointed out the existence of a population of compact, massive galaxies at $z \sim 2.5$ having effective radii and high stellar mass surface densities. It is possible that these are the result of massive and concentrated starbursts in highly dissipative, gas-rich mergers at high redshift $(z \sim 3-6)$. Extrapolation of our results to higher redshifts suggests that very dense stellar "cores" are indeed actively being formed in the LBG population.

\subsubsection{Prediction for the Faint-End Slope of the LBG Luminosity Function}

The UV luminosity function (LF) has now been evaluated over the entire redshift range from $z=0$ to 6 . Interestingly, the faint-end slope of the LF has been found to flatten with decreasing redshift from $\alpha \sim 1.74$ at $z=6$ to $\sim 1.2$ at $z=0$ (Yan \& Windhorst 2004a, 2004b; Wyder et al. 2005; Bouwens et al. 2007; Ryan et al. 2007). Various simulations and models have been tried to reproduce the LF and explain its flattening at faint magnitudes (e.g., Night et al. 2006; Finlator et al. 2007; Khochfar et al. 2007). As shown below, the existence of compact starbursts in LBGs may offer a natural explanation for the steep slopes that are observed at high redshifts. The LF of star-forming regions is well known to have a slope of $\alpha \approx 2$ over a wide range in physical scales from $\mathrm{H}$ in regions to SSCs and beyond (e.g., Kennicutt et al. 1989; Meurer et al. 1995; Elmegreen \& Efremov 1997; Zepf et al. 1999; Alonso-Herrero et al. 2002; Bradley et al. 2006 and references therein).

We consider the following, very simple, toy model. Let $N(L, z)$ be the total number density of galaxies with UV luminosity between $L$ and $L+d L$ and redshift between $z$ and $z+d z$. We also assume that the stellar IMF, as well as the star formation efficiency in galaxies, does not change with redshift. The UV luminosity of an individual galaxy at a given time $t$ is the sum over the luminosity of all its previous star formation events, $L_{i}$, at that time:

$$
L_{\mathrm{LBG}}^{\mathrm{UV}}(t)=\sum_{i} L_{i}(t)
$$

The luminosity arising from an "event" can take any arbitrary form (e.g., burst, constant, declining), and it is not important what 
caused the event. For LBGs, it is instructive to make a distinction between the contribution to the total luminosity from a series of $N$ (semi)discrete starbursts, $L_{\text {burst }}^{i}$, and that due to a diffuse starforming component that is slowly evolving with time, $L_{\text {diffuse, }}$, so that

$$
L_{\mathrm{LBG}}^{\mathrm{UV}}(t)=L_{\text {diffuse }}(t)+\sum_{i=1}^{N} L_{\text {burst }}^{i}(t)
$$

We have learned that the UV luminosity from an LBG is typically dominated by a series of $N_{\text {knot }}$ knots $(\sim 1-2 \mathrm{kpc}$ in size) and that each knot can be further resolved into a series of $N_{\mathrm{SSB}}$ SSBs $(\sim 100-300 \mathrm{pc}$ in size $)$, and thus

$$
L_{\mathrm{LBG}}^{\mathrm{UV}}(t)=L_{\text {diffuse }}(t)+\sum_{i}^{N_{\mathrm{knot}}} \sum_{j}^{N_{\mathrm{SSB}}} L_{\mathrm{SSB}}^{i, j}(t),
$$

where $L_{\mathrm{SSB}}^{i, j}(t)$ is the luminosity of an SSB region $j$ in knot $i$ at time $t$. For (proto)galaxies that are just undergoing their first episode of massive star formation the above expression will simplify enormously, as we can neglect the diffuse component, $L_{\text {diffuse }}(t)$, and both $N_{\text {knot }}$ and $N_{\text {SSB }}$ will be near unity. The most simple case is represented by $N_{\mathrm{knot}}=N_{\mathrm{SSB}}=1$, as observed in some of our local LBG analogs, and (at least in this extreme case) the total UV LF will be purely determined by a random sampling of the LF of the SSBs over the galaxy population in a given redshift interval. As shown by Meurer et al. (1995), the "anatomy of starbursts" typically consists of irregularly shaped cloud(s) (SSBs) with embedded compact sources (SSCs), the brightest of which are massive forming (globular) clusters. Star formation thus occurs in hierarchically clustered systems ranging from subparsec to multikiloparsec scales and is believed to be generated at all scales by self-gravity and turbulence within an underlying hierarchical gas mass distribution $n(M) d M \propto M^{-\alpha} d M$ (Elmegreen \& Efremov 1996, 1997 and references therein). Because the UV luminosity of starbursts is dominated by its OB stars at all scales, on average $M \propto L$ with $\alpha \sim 1.7-2$ for the references given above. Therefore, in the regime where the LF is dominated by young galaxies generating their first significant amount of stellar mass, i.e., at relatively faint magnitudes and high redshift, our simple model predicts that the galaxy LF observed will essentially be a random subsampling of the LF of starburst regions, thereby maintaining its intrinsic slope. It is interesting to note that, for example, the faintest $B_{435^{-}}, V_{606^{-}}$, or $i_{775^{-}}$dropout galaxies observed at $z \sim 4-6$ have stellar masses very similar to those of our starburst regions $\left(\sim 10^{8}-10^{9} M_{\odot}\right)$.

Generally, it will be impossible to model the full UV LF quantitatively in this way (e.g., eq. [4]) without resorting to semianalytic models or simulations because it involves the convolution of large numbers of galaxies with a range of star formation histories including effects such as merging, feedback, and dust. However, qualitatively one expects that, when all processes are considered, the slope will flatten from its initial value. Thus, we expect that the faint-end slope of the LF at least steepens with redshift and will deviate less and less from the "pure" star formation LF with a slope of $\alpha \approx 2$, exactly as observed.

\section{CONCLUSIONS}

The study of galaxies in the early universe can benefit enormously from the study of objects that are relatively nearby, provided that suitable samples of local analogs can be found. H05 and H07 selected "supercompact," UV-luminous galaxies in a large GALEX-SDSS cross matched sample (see $\S 1$ ). Because these objects match LBGs in terms of size, SFR, surface brightness, mass, metallicity, kinematics, dust, and UV-optical color, this sample of local LBG analogs is well suited for investigating the connection between star formation and morphology at a level of detail and precision that is impossible for high-redshift galaxies. Emission-line diagnostics indicate that they are dominated by starbursts and that the line ratios show small offsets with respect to the local star-forming population analogous to those observed for high-redshift LBGs ( $\S 3.2)$.

In this paper we present $H S T$ imaging data in $U_{330}, z_{850}$, and $\mathrm{H} \alpha$ of a subsample of eight local LBG analogs in order to investigate their morphologies. The effective radii estimated from the $U_{330}$ images range from unresolved ( $\left.\lesssim 100 \mathrm{pc}\right)$ to $\sim 2 \mathrm{kpc}$, confirming that the objects are highly compact. The objects are slightly more extended in $z_{850}(\sim 1-2 \mathrm{kpc})$, and they generally have color gradients with relatively blue inner colors and redder outer colors. These gradients are due to the fact that the starburst regions are very compact and are embedded in an older, more extended population ( $\S \S 3.3$ and 3.4). The internal extinctions amount to $E(B-$ $V) \approx 0.01-0.14 \mathrm{mag}$ based on the bolometric dust-to-FUV luminosity ratios observed with Spitzer and GALEX (§ 3.5). We have calculated the morphological parameters $G, M_{20}$, and $C$ and find that local LBG analogs have irregular morphologies that, on average, lie in between those expected for major mergers, disks, and bulges. We have simulated our data at the depth and resolution of LBGs at $z=[1.5,3.0,4.0]$ in the COSMOS, GOODS, and UDF surveys, and we have carried out direct comparisons with the morphologies of $z \sim 1.5$ star-forming galaxies and $z \sim 4$ LBGs extracted from these surveys. The morphological parameters measured for the artificially redshifted sample are very similar to those obtained for the distant LBGs. This establishes that local LBG analogs and high-redshift LBGs are likely to be in a similar phase of their evolution ( $\S 3.6)$. The UVLGs are more typical of LBGs in comparison with two very nearby LBG analogs, Haro 11 and VV 114 at $z=0.02$. The latter two are more similar to the "clump cluster galaxies" due to their multiple nuclei at relatively large separation (Elmegreen et al. 2007). Although more local LBG analogs of this type may be found as well, we note that our current sample is biased against finding such objects $(\S 3.6 .4)$.

Although a large fraction of LBGs at $z \sim 3-4$ is consistent with having exponential or $r^{1 / 4}$ light profiles, their visual morphologies include multiple, double, and perturbed systems. Morphological parameters indicate that the majority of LBGs are likely to be in a range of stages associated with minor and major galaxy merging (e.g., Giavalisco et al. 1996b; Lotz et al. 2006; Ravindranath et al. 2006; Elmegreen et al. 2007). In contrast, Burgarella et al. (2006) find that the majority of luminous, UV-selected starforming galaxies at $z \sim 1$ have a disk or spiral morphology. At a similar redshift, but at much lower UV luminosities, de Mello et al. (2006) find a mix of morphological types that include early-type galaxies and starbursts. We note that this is a direct consequence of the selection of galaxies purely based on their $\mathrm{UV}$ emission. As discussed here and by $\mathrm{H} 05$, at relatively low redshifts $(z \sim 0-1)$ the construction of a sample of galaxies that most closely resembles high-redshift LBGs in terms of their physical properties requires additional selection criteria (e.g., UV surface brightness) rather than selection on UV luminosity or the presence of a "Lyman break" alone.

We have carried out a detailed investigation of the small-scale structure of the local LBG analogs in terms of their star-forming regions $(\S 4)$. The starburst regions observed in the local LBG analogs are highly compact (radii of 100-300 pc) and characterized 
by bright, unresolved knots of emission within a larger region of diffuse star formation that extends up to a few kiloparsecs in size. The total UV emission from these compact SSBs is substantial, indicating that $30 \%-80 \%$ of the total SFR is generated in these knots. Comparison with Starburst 99 evolutionary tracks indicates that some of the regions are due to very young ( $\sim 6 \mathrm{Myr})$ and massive $\left(\sim 10^{8} M_{\odot}\right)$ bursts, while other regions may have been forming stars in a more continuous manner for several tens to several hundreds of Myr as indicated by their relatively red colors and high $\mathrm{H} \alpha$ equivalent widths. The SSBs are likely to be a blend of diffuse star-forming regions, stellar associations, and super or globular star clusters, as demonstrated by comparing the images of Haro 11 and VV 114 at $z=0.02$ to the same images redshifted to $z=0.15-4.0$. The SSBs are generally embedded in a diffuse, older component having ages ranging from a few Gyr to a Hubble time, depending on the star formation history. In this respect, the precursors of the UVLGs may have been more evolved than those of LBGs at high redshift (although the presence of a "maximally old" stellar component in LBGs is consistent with the observations).

Although some of the SDSS images showed limited evidence for close companions or faint extended emission, the optical HST images clearly reveal disturbed morphologies at scales well below the SDSS seeing or sensitivity. Each object shows evidence of merging or interactions, such as multiplicity of position angles, tidal debris or tails, or close companions. Most morphological information about high-redshift galaxies has been derived from rest-frame UV images. In our sample, the $U_{330}$ band does not establish a definite case for a merger in any single one of our galaxies. The disturbed optical ( $z$ band) morphologies, together with the luminous, compact SSBs, suggest that mergers of relatively gas-rich objects trigger vigorous episodes of star formation, with general properties reminiscent of simulations of collisional starbursts $(\S 5.1)$. Our results on local LBGs and their similarity to their high- $z$ LBGs constitute the most direct evidence to date that the onset of star formation and morphological structures of highredshift LBGs are largely driven by highly dissipational merging.

We discuss several implications of our results for the interpretation of lower resolution data on high-redshift LBGs. Resolution effects will cause the compact starburst regions to blend into typically one or two bright LBG-like knots. Although these knots will appear relatively featureless with total half-light radii of 1-2 kpc, the substructure of our LBG analogs indicates that they should generally not be interpreted as being evidence of bulges or disks. Evidence that the substructure of high-redshift LBGs is indeed dominated by SSBs is found in a few cases where LBGs are highly magnified by gravitational lensing ( $\S 5.2 .1$ ). However, the strong relation we find between merging and the triggering of very compact, massive SSBs indicates that these events may be closely linked to the formation of stellar bulges. Furthermore, we suggest that the prominence of luminous, unresolved (super) starburst regions in forming galaxies may provide a natural explanation for the value of the faint-end slope of the UV LF at high redshift by an extension of the local star-forming region LF, which is well known to have a power-law slope of $\alpha \approx 2(\S 5.2 .2)$.

Future work.- - In Paper II we will study a significantly larger sample of local LBG analogs to be observed with HST in Cycle 16 in the FUV/optical using the ACS/SBC and WFPC2. Follow-up spectroscopy is being used to study emission-line ratios and kinematics, measure outflows, and identify companion objects. The ongoing surveys with GALEX will provide larger samples of local LBGs that will be used to derive better sample statistics.

This paper has benefited from discussions and helpful comments from numerous friends and colleagues. We thank Casey Papovich, Masami Ouchi, and Isa Oliveira for carefully reading through the manuscript. We further thank Rychard Bouwens, Nick Cross, Ricardo Demarco, Marijn Franx, Lisa Kewley, Cheng Li, Crystal Martin, Alessandro Rettura, Samir Salim, Christi Tremonti, Arjen van der Wel, and Andrew Zirm for discussion of various parts of this paper. R. A. O. thanks Gabrelle Saurage for her excellent support during observations at APO.

Based on observations made with the NASA/ESA Hubble Space Telescope, which is operated by the Association of Universities for Research in Astronomy, Inc., under NASA contract NAS5-26555. These observations are associated with program 10920. Based on observations obtained with the Apache Point Observatory (APO) $3.5 \mathrm{~m}$ telescope, which is owned and operated by the Astrophysical Research Consortium.

\section{APPENDIX}

\section{SCARPA ET AL. (2007) REVISITED}

In a recent paper, Scarpa et al. (2007) have tried to argue that the sample of compact UVLGs selected by H05 and H07 are not true analogs of LBGs. Scarpa et al. (2007) claim that the sizes estimated from the SDSS $u$-band images grossly underestimate the actual size in the UV and that the UVLGs therefore are much larger and lower in surface brightness than LBGs. Here we briefly show that this claim is based on the combination of a faulty analysis and incorrect assumptions, which are easily refuted by the HST data presented in this paper.

Scarpa et al. (2007) claim that it is only possible to detect central bulges in the SDSS $u$-band images and that fainter, extended disks would be missed. They reach this conclusion by fitting the SDSS $r$-band images with a two-component model (point source plus disk) and then using the half-light radius of only the disk component to recompute the size of the UVLGs. In doing this, the authors made a serious mistake because they ignore the fact that, on average, half the light in their model fits comes from the central "point source" (see their Table 1).

The claim in the Scarpa et al. (2007) paper that most of the FUV flux in compact UVLGs should come from a large disk component relies on the observation that there exists a population of galaxies with relatively red bulges not visible in the FUV and very blue outer rings or disks that are only apparent in the UV (e.g., Thilker et al. 2005). It should be clear both from the results presented in H05 and H07 and from the analysis of HST data presented here that UVLGs are an entirely different class of objects. In fact, the UVLGs are clearly more extended in the optical than in the UV due to an underlying (older) component. The UVLGs have very blue inner colors and become redder outward (see Fig. 4 and Table 4). This is opposite to the assumption of Scarpa et al. (2007).

The HST $U_{330}$ images confirm that the UVLGs are indeed very compact objects. In fact, in most cases, the half-light radius is even smaller than the seeing-deconvolved SDSS $u$-band size (Table 4). In the case of both J005527.46-002148.7 and J080844.26+394852.3 the UV flux is dominated by an unresolved component even at the HST resolution. Furthermore, when we extrapolate the slope of the UV 
continuum measured from the FUV - NUV color and predict the $U_{330}$ flux, we find good agreement with the measured $U_{330}$ flux $(\S 3.3)$, indicating that the $U_{330}$ (and SDSS $u$ band) image is a good tracer of the FUV morphology. In the case of J214500.25+011157.3 we have an ACS/SBC image taken through F150LP, a filter that is almost identical to the GALEX FUV filter (see Teplitz et al. 2006). The corresponding FUV surface brightness calculated solely from the ACS data, $\log _{10} I_{\mathrm{FUV} \text {, ACS }}=9.53 L_{\odot} \mathrm{kpc}^{-2}$, is slightly lower than the value of $\mathrm{H} 07$ based on GALEX and SDSS $\left(\log _{10} I_{\mathrm{FUV}}=9.82 L_{\odot} \mathrm{kpc}^{-2}\right)$, but still well above our LBG selection threshold of $\log _{10} I_{\mathrm{FUV}}=9 L_{\odot} \mathrm{kpc}^{-2}$.

As we have discussed above, the galaxy sizes defined by Scarpa et al. (2007) pertain only to the disk component and thus cannot be compared with the half-light radii quoted by H05 and H07. Scarpa et al. (2007) also did not apply their profile fitting methodology to the full sample in a uniform way. They only applied their analysis to the objects that were marked as "supercompact" by H07 and not to the other UVLGs. As a result, their concluding Figure 5 is misleading. It appears to show that the LBG analogs have a similar FUV surface brightness as the main sample. Had they applied their technique to all the objects in the sample, the compact UVLGs would still form an upper envelope to the $I_{\mathrm{FUV}}$ distribution, with the overall $I_{\mathrm{FUV}}$ distribution shifted downward toward lower surface brightness due to the fact that the point-source flux in their fits was ignored.

Finally, we would like to remind the reader that the real test of whether or not a given sample of nearby galaxies are good analogs of LBGs is not the UV selection per se (note that the LBG LF extends over many orders of magnitude; e.g., Bouwens et al. 2007), but whether their main physical properties are indeed similar. We firmly believe that our sample of LBG analogs have passed this test.

\section{REFERENCES}

Abraham, R. G., van den Bergh, S., Glazebrook, K., Ellis, R. S., Santiago, B. X. Surma, P., \& Griffiths, R. E. 1996, ApJS, 107, 1

Adelberger, K. L., \& Steidel, C. C. 2000, ApJ, 544, 218

Adelberger, K. L., Steidel, C. C., Pettini, M., Shapley, A. E., Reddy, N. A., \& Erb, D. K. 2005, ApJ, 619, 697

Adelberger, K. L., Steidel, C. C., Shapley, A. E., Hunt, M. P., Erb, D. K., Reddy, N. A., \& Pettini, M. 2004, ApJ, 607, 226

Alonso-Herrero, A., Rieke, G. H., Rieke, M. J., \& Scoville, N. Z. 2002, AJ, 124,166

Basu-Zych, A., et al. 2007, ApJS, 173, 457

Bekki, K. 2004, Publ. Astron. Soc. Australia, 21, 167

Bekki, K., \& Couch, W. J. 2001, ApJ, 557, L19

Bergvall, N., \& Östlin, G. 2002, A\&A, 390, 891

Bergvall, N., Zackrisson, E., Andersson, B.-G., Arnberg, D., Masegosa, J., \& Oumlstlin, G. 2006, A\&A, 448, 513

Bertin, E., \& Arnouts, S. 1996, A\&AS, 117, 393

Bouwens, R., Broadhurst, T., \& Silk, J. 1998, ApJ, 506, 557

Bouwens, R. J., Illingworth, G. D., Blakeslee, J. P., Broadhurst, T. J., \& Franx, M. 2004, ApJ, 611, L1

Bouwens, R. J., Illingworth, G. D., Blakeslee, J. P., \& Franx, M. 2006, ApJ, 653,53

Bouwens, R. J., Illingworth, G. D., Franx, M., \& Ford, H. 2007, ApJ, 670, 928

Bradley, T. R., Knapen, J. H., Beckman, J. E., \& Folkes, S. L. 2006, A\&A, 459, L13

Bunker, A. J., Moustakas, L. A., \& Davis, M. 2000, ApJ, 531, 95

Burgarella, D., Buat, V., \& Iglesias-Páramo, J. 2005, MNRAS, 360, 1413

Burgarella, D., et al. 2006, A\&A, 450, 69

Calzetti, D. 2001, PASP, 113, 1449

Chapman, S. C., Blain, A. W., Smail, I., \& Ivison, R. J. 2005, ApJ, 622, 772

Conselice, C. J. 2003, ApJS, 147, 1 2006, ApJ, 638, 686

Conselice, C. J., et al. 2004, ApJ, 600, L139

Conti, P. S. 1991, ApJ, 377, 115

Daddi, E., et al. 2005, ApJ, 631, L13

Dahlen, T., Mobasher, B., Dickinson, M., Ferguson, H. C., Giavalisco, M., Kretchmer, C., \& Ravindranath, S. 2007, ApJ, 654, 172

Dale, D. A., \& Helou, G. 2002, ApJ, 576, 159

Dale, D. A., et al. 2005, ApJ, 633, 857

de Mello, D. F., Wadadekar, Y., Dahlen, T., Casertano, S., \& Gardner, J. P. 2006, AJ, 131, 216

di Matteo, P., Combes, F., Melchior, A.-L., \& Semelin, B. 2007, A\&A, 468, 61

Ellis, R., Santos, M. R., Kneib, J.-P., \& Kuijken, K. 2001, ApJ, 560, L119

Elmegreen, B. G., \& Efremov, Y. N. 1996, ApJ, 466, 802 1997, ApJ, 480, 235

Elmegreen, D. M., Elmegreen, B. G., Ravindranath, S., \& Coe, D. A. 2007, ApJ, 658, 763

Elmegreen, D. M., Elmegreen, B. G., \& Sheets, C. M. 2004, ApJ, 603, 74

Erb, D. K., Shapley, A. E., Pettini, M., Steidel, C. C., Reddy, N. A., \& Adelberger, K. L. 2006a, ApJ, 644, 813

Erb, D. K., Shapley, A. E., Steidel, C. C., Pettini, M., Adelberger, K. L., Hunt, M. P., Moorwood, A. F. M., \& Cuby, J.-G. 2003, ApJ, 591, 101

Erb, D. K., Steidel, C. C., Shapley, A. E., Pettini, M., Reddy, N. A., \& Adelberger, K. L. 2006b, ApJ, 647, 128

Fanelli, M. N., O'Connell, R. W., \& Thuan, T. X. 1988, ApJ, 334, 665

Ferguson, H. C., et al. 2004, ApJ, 600, L107
Finlator, K., Davé, R., \& Oppenheimer, B. D. 2007, MNRAS, 376, 1861

Förster Schreiber, N. M., et al. 2006, ApJ, 645, 1062

Franx, M., Illingworth, G. D., Kelson, D. D., van Dokkum, P. G., \& Tran, K.-V. 1997, ApJ, 486, L75

Genzel, R., et al. 2006, Nature, 442, 786

Giavalisco, M., \& Dickinson, M. 2001, ApJ, 550, 177

Giavalisco, M., Livio, M., Bohlin, R. C., Macchetto, F. D., \& Stecher, T. P. 1996a, AJ, 112, 369

Giavalisco, M., Steidel, C. C., \& Macchetto, F. D. 1996b, ApJ, 470, 189

Giavalisco, M., et al. 2004, ApJ, 600, L103

Gil de Paz, A., Madore, B. F., \& Pevunova, O. 2003, ApJS, 147, 29

Goldader, J. D., Meurer, G., Heckman, T. M., Seibert, M., Sanders, D. B., Calzetti, D., \& Steidel, C. C. 2002, ApJ, 568, 651

Governato, F., Ghigna, S., Moore, B., Quinn, T., Stadel, J., \& Lake, G. 2001, ApJ, 547, 555

Grimes, J. P., Heckman, T., Hoopes, C., Strickland, D., Aloisi, A., Meurer, G., \& Ptak, A. 2006, ApJ, 648, 310

Grimes, J. P., et al. 2007, ApJ, 668, 891

Hayes, M., Ostlin, G., Atek, H., Kunth, D., Mas-Hesse, J. M., Leitherer, C., Jimenez-Bailon, E., \& Adamo, A. 2007, MNRAS, 382, 1465

Heckman, T. M., et al. 2005, ApJ, 619, L35 (H05)

Hibbard, J. E., \& Vacca, W. D. 1997, AJ, 114, 1741

Hoopes, C., et al. 2007, ApJS, 173, 441 (H07)

Huang, J.-S., et al. 2005, ApJ, 634, 137

Hunter, D. A., \& Hoffman, L. 1999, AJ, 117, 2789

Immeli, A., Samland, M., Westera, P., \& Gerhard, O. 2004, ApJ, 611, 20

Izotov, Y. I., Foltz, C. B., Green, R. F., Guseva, N. G., \& Thuan, T. X. 1997, ApJ, 487, L37

Kauffmann, G., et al. 2003, MNRAS, 346, 1055

Kennicutt, R. C., Jr. 1998, ARA\&A, 36, 189

Kennicutt, R. C., Jr., Edgar, B. K., \& Hodge, P. W. 1989, ApJ, 337, 761

Kewley, L. J., Dopita, M. A., Sutherland, R. S., Heisler, C. A., \& Trevena, J. 2001, ApJ, 556, 121

Kewley, L. J., Groves, B., Kauffmann, G., \& Heckman, T. 2006, MNRAS, 372 , 961

Khochfar, S., Silk, J., Windhorst, R. A., \& Ryan, R. E., Jr. 2007, ApJ, 668, L115

Knop, R. A., Soifer, B. T., Graham, J. R., Matthews, K., Sanders, D. B., \& Scoville, N. Z. 1994, AJ, 107, 920

Koekemoer, A. M., Fruchter, A. S., Hook, R. N., \& Hack, W. 2002, in The 2002 HST Calibration Workshop: Hubble after the Installation of the ACS and the NICMOS Cooling System, ed. S. Arribas, A. Koekemoer, \& B. Whitmore (Baltimore: STScI), 337

Kong, X., Charlot, S., Brinchmann, J., \& Fall, S. M. 2004, MNRAS, 349, 769

Kunth, D., Leitherer, C., Mas-Hesse, J. M., Östlin, G., \& Petrosian, A. 2003, ApJ, 597, 263

Lambas, D. G., Tissera, P. B., Alonso, M. S., \& Coldwell, G. 2003, MNRAS, 346,1189

Larson, R. B., \& Tinsley, B. M. 1978, ApJ, 219, 46

Law, D. R., Steidel, C. C., Erb, D. K., Larkin, J. E., Pettini, M., Shapley, A. E., \& Wright, S. A. 2007a, ApJ, 669, 929

Law, D. R., Steidel, C. C., Erb, D. K., Pettini, M., Reddy, N. A., Shapley, A. E., Adelberger, K. L., \& Simenc, D. J. 2007b, ApJ, 656, 1

Leitherer, C., \& Heckman, T. M. 1995, ApJS, 96, 9

Leitherer, C., et al. 1999, ApJS, 123, 3 
Li, C., Kauffmann, G., Heckman, T., Jing, Y. P., \& White, S. D. M. 2008, MNRAS, in press (arXiv:0711.3792)

Lilly, S. J., Le Fevre, O., Hammer, F., \& Crampton, D. 1996, ApJ, 460, L1

Lotz, J. M., Madau, P., Giavalisco, M., Primack, J., \& Ferguson, H. C. 2006, ApJ, 636, 592

Lotz, J. M., Primack, J., \& Madau, P. 2004, AJ, 128, 163

Lowenthal, J. D., et al. 1997, ApJ, 481, 673

Martin, D. C., et al. 2005, ApJ, 619, L1

Meurer, G. R., Heckman, T. M., Lehnert, M. D., Leitherer, C., \& Lowenthal, J. 1997, AJ, 114, 54

Meurer, G. R., Heckman, T. M., Leitherer, C., Kinney, A., Robert, C., \& Garnett, D. R. 1995, AJ, 110, 2665

Mihos, J. C., \& Hernquist, L. 1994a, ApJ, 425, L13 1994b, ApJ, 431, L9

Moustakas, L. A., \& Somerville, R. S. 2002, ApJ, 577, 1

Night, C., Nagamine, K., Springel, V., \& Hernquist, L. 2006, MNRAS, 366, 705

Nikolic, B., Cullen, H., \& Alexander, P. 2004, MNRAS, 355, 874

Noeske, K. G., Koo, D. C., Phillips, A. C., Willmer, C. N. A., Melbourne, J., Gil de Paz, A., \& Papaderos, P. 2006, ApJ, 640, L143

Noguchi, M. 1999, ApJ, 514, 77

Östlin, G., Bergvall, N., \& Roennback, J. 1998, A\&A, 335, 85

Ouchi, M., et al. 2004a, ApJ, 611, 660

$2004 \mathrm{~b}$, ApJ, 611, 685

2008, ApJS, in press (arXiv:0707.3161)

Overzier, R. A., et al. 2008, ApJ, 673, 143

Owers, M., Blake, C., Couch, W., Pracy, M., \& Bekki, K. 2007, MNRAS, 381, 494

Papovich, C., Dickinson, M., \& Ferguson, H. C. 2001, ApJ, 559, 620

Papovich, C., Dickinson, M., Giavalisco, M., Conselice, C. J., \& Ferguson, H. C. 2005, ApJ, 631, 101

Papovich, C., Giavalisco, M., Dickinson, M., Conselice, C. J., \& Ferguson, H. C. 2003, ApJ, 598, 827

Ravindranath, S., et al. 2006, ApJ, 652, 963

Reddy, N. A., Steidel, C. C., Fadda, D., Yan, L., Pettini, M., Shapley, A. E., Erb, D. K., \& Adelberger, K. L. 2006, ApJ, 644, 792

Ryan, R. E., Jr., et al. 2007, ApJ, 668, 839

Salim, S., et al. 2007, ApJS, 173, 267

Sanders, D. B., \& Mirabel, I. F. 1996, ARA\&A, 34, 749

Sargent, W. L. W., \& Filippenko, A. V. 1991, AJ, 102, 107

Scarpa, R., Falomo, R., \& Lerner, E. 2007, ApJ, 668, 74
Schiminovich, D., et al. 2005, ApJ, 619, L47

Schlegel, D. J., Finkbeiner, D. P., \& Davis, M. 1998, ApJ, 500, 525

Scoville, N. Z., et al. 2000, AJ, 119, 991

Seibert, M., et al. 2005, ApJ, 619, L55

Shapley, A. E., Coil, A. L., Ma, C.-P., \& Bundy, K. 2005, ApJ, 635, 1006

Shapley, A. E., Steidel, C. C., Adelberger, K. L., Dickinson, M., Giavalisco, M., \& Pettini, M. 2001, ApJ, 562, 95

Shaya, E. J., Dowling, D. M., Currie, D. G., Faber, S. M., \& Groth, E. J. 1994, AJ, 107, 1675

Sirianni, M., et al. 2005, PASP, 117, 1049

Smail, I., et al. 2007, ApJ, 654, L33

Somerville, R. S., Primack, J. R., \& Faber, S. M. 2001, MNRAS, 320, 504

Springel, V., \& Hernquist, L. 2005, ApJ, 622, L9

Steidel, C. C., Adelberger, K. L., Giavalisco, M., Dickinson, M., \& Pettini, M. 1999, ApJ, 519, 1

Steidel, C. C., Giavalisco, M., Pettini, M., Dickinson, M., \& Adelberger, K. L. 1996, ApJ, 462, L17

Steidel, C. C., \& Hamilton, D. 1993, AJ, 105, 2017

Swinbank, A. M., Bower, R. G., Smith, G. P., Wilman, R. J., Smail, I., Ellis, R. S.,

Morris, S. L., \& Kneib, J.-P. 2007, MNRAS, 376, 479

Takeuchi, T. T., Buat, V., \& Burgarella, D. 2005, A\&A, 440, L17

Telles, E., \& Terlevich, R. 1997, MNRAS, 286, 183

Teplitz, H. I., et al. 2006, AJ, 132, 853

Thilker, D. A., et al. 2005, ApJ, 619, L79

van Dokkum, P. G., et al. 2006, ApJ, 638, L59

Vázquez, G. A., \& Leitherer, C. 2005, ApJ, 621, 695

Whitmore, B. C., Zhang, Q., Leitherer, C., Fall, S. M., Schweizer, F., \& Miller, B. W. 1999, AJ, 118, 1551

Wong, O. I., et al. 2006, MNRAS, 370, 1607

Woods, D. F., Geller, M. J., \& Barton, E. J. 2006, AJ, 132, 197

Wright, S. A., et al. 2007, ApJ, 658, 78

Wyder, T. K., et al. 2005, ApJ, 619, L15

Yan, H., \& Windhorst, R. A. 2004a, ApJ, 600, L1 2004b, ApJ, 612, L93

York, D. G., et al. 2000, AJ, 120, 1579

Yoshida, M., et al. 2006, ApJ, 653, 988

Younger, J. D., et al. 2007, ApJ, 671, 1241

Zepf, S. E., Ashman, K. M., English, J., Freeman, K. C., \& Sharples, R. M. 1999, AJ, 118, 752

Zirm, A. W., et al. 2007, ApJ, 656, 66 